\title{
RF microplasmas with energies suited to in situ selective cleaning of surface adsorbates in ion microtraps
}

\author{
Mariam Akhtar ${ }^{1,2}$, Guido Wilpers ${ }^{1}$, Kaushal Choonee ${ }^{1}$, \\ Erling Riis ${ }^{2}$ and Alastair G. Sinclair ${ }^{1}$ \\ ${ }^{1}$ National Physical Laboratory, Teddington, TW11 0LW, UK \\ ${ }^{2}$ University of Strathclyde, Glasgow, G4 0NG, UK \\ E-mail: alastair.sinclair@npl.co.uk
}

\begin{abstract}
.
We have demonstrated a capacitively-coupled, RF microplasma inside the 3D electrode structure of an ion microtrap device. For this work, devices with an inter-electrode distance of $340 \mu \mathrm{m}$ were used. The microplasmas were operated at $\Omega_{R F} / 2 \pi=23 \mathrm{MHz}$, in both $\mathrm{He}$ and $\mathrm{He}_{2} \mathrm{~N}_{2}$ gas mixtures, over a range of RF amplitudes (140 V to $220 \mathrm{~V}$ ) and pressures (250 mbar to $910 \mathrm{mbar}$ ). Spectroscopic analysis of the He I $667 \mathrm{~nm}$ and $\mathrm{H} \alpha 656 \mathrm{~nm}$ emission lines yielded the gas temperature and electron density, which enabled calculation of the mean ion bombardment energy. For the range of operating parameters studied, we calculated mean $\mathrm{He}^{+}$energies to be between $0.3 \mathrm{eV}$ and $4.1 \mathrm{eV}$. While these energies are less than the threshold for He sputtering of hydrocarbon adsorbates on $\mathrm{Au}$, we calculate that the high energy tail of the distribution should remove adsorbate monolayers in as little as 1 minute of processing. We also calculate that the distribution is insufficiently energetic to have any significant effect on the $\mathrm{Au}$ electrode surface within that duration. Our results suggest that the microplasma technique is suited to in situ selective removal of surface adsorbates from ion microtrap electrodes.
\end{abstract}

Keywords: ion trap, microplasma, microdischarge, motional decoherence, spectroscopy 


\section{Introduction}

Trapped atomic ions are an important physical system for investigating quantum information processing [1], quantum simulation [2], quantum metrology [3], and optical atomic clocks [4]. The ability to store and cool ions, virtually isolated from the surrounding environment, has been essential for taking advantage of their quantum behaviour [5]. In the aforementioned applications, several sources of decoherence can hinder high-fidelity coherent control of the ions' quantum state; one of the foremost is motional decoherence [6-9]. Minimising this to negligible levels in microfabricated ion traps is paramount for development of quantum technologies based on trapped ions. Motional decoherence is due to fluctuating electric fields $[6,8,10]$, however the exact origin of this electric field noise is still not well understood.

Ions in microfabricated traps [7], where the ionelectrode distance $(d)$ is typically in the range $80 \mu \mathrm{m} \leqslant$ $d \leqslant 250 \mu \mathrm{m}$, are particularly sensitive to this noise, which has been found to scale as $\sim 1 / d^{4}[6,8,11,12]$. Moreover, resultant heating rates are routinely well above that which would be expected from Johnson noise, giving rise to the term "anomalous heating" [8]. For example at $d \leqslant 365 \mu \mathrm{m}$, ion heating rates in room-temperature traps have been shown to be more than three orders of magnitude above the value expected from Johnson noise $[8,13]$. Cooling of trap electrodes with liquid $\mathrm{N}_{2}$ to $150 \mathrm{~K}$ [9], and with liquid He to $6 \mathrm{~K}$ [14], was observed to reduce room-temperature heating rates by 1 and 7 orders of magnitude respectively. However even at low temperatures the motional heating rate is still notably above the level due to Johnson noise alone. For example, in $[14,15]$ the measured heating rates at cryogenic temperatures were still 3 orders of magnitude greater than the value expected from Johnson noise.

Models have shown that the observed scaling of heating rates with distance can be explained by fluctuating patch potentials $[6,8]$ or by thin dielectric layers (i.e. native oxide or hydrocarbons) on electrodes [10]. The latest experimental results $[12,16]$ support the latter model. It is noted that a clean $\mathrm{Au}$ surface exposed to air would acquire 0.4 monolayers of hydrocarbon coverage within a few minutes, and that a vacuum-bake procedure can increase coverage to a few monolayers [10]. In addition, ion microtraps of planar electrode geometry have been used to investigate the influence of electrode surface composition on trapped ion heating rate. A correlation was observed between the removal of carbon adsorbed on electrode surfaces, and a hundred-fold reduction of the heating rate $[17,18]$. A further investigation concerned noise due to carbon adatom diffusion on $\mathrm{Au}$ surfaces [19], which showed a model for electric field noise in line with measurements.

We have developed monolithic ion microtrap devices which are in the form of a linear, segmented trapping array [20-22]. These devices have a 3D electrode structure which maximises the depth and harmonicity of the trapping potential. However, this 3D structure also makes it inappropriate for treatment of $\mathrm{Au}$ electrode surfaces by the ion beam method discussed above. In addition to removing contaminants, an energetic beam (e.g. with $\mathrm{Ar}^{+}$ energies of $300-2000 \mathrm{eV}[17,18])$ will also remove $\mathrm{Au}$ electrode material since the sputtering threshold for $\mathrm{Au}$ with $\mathrm{Ar}^{+}$is $15 \mathrm{eV}$ [23]. Redeposition of $\mathrm{Au}$ on dielectric surfaces, intended as insulating gaps in the electrode structure, risks the creation of an electrical short thus failing the device.

In contrast, a gentler approach to surface processing has been investigated previously by introducing a coil near the surface of the trap to generate an $\mathrm{Ar}$ inductively-coupled plasma [24]. The ion bombardment energies produced were approximately $20 \mathrm{eV}$, which should be compared to $30 \mathrm{eV}$ threshold for sputtering the $\mathrm{Nb}$ electrodes of the trap in question. Therefore, the process is more likely to be selective in the sputtering of only hydrocarbon material from the electrode surface. Using this method, a four-fold reduction in heating rate was reported. We adopt an alternative approach which avoids the need for an inductive coil in the system. Instead, we generate a capacitivelycoupled, RF microplasma by using the ion trap electrodes themselves. We show this to be suited to in situ, low-energy, selective sputtering of only the hydrocarbon contaminants on electrode surfaces, and not the $\mathrm{Au}$ of the electrodes themselves. This could be a useful technique in future studies of electric field noise, for separating the effects of surface cleanliness from any due to electrode surface order [25].

This paper presents measurements to assess the suitability of using a microplasma for selective sputtering of adsorbed hydrocarbon contaminants from electrode surfaces in an ion microtrap device. In order to do this, the mean ion bombardment energy $\left(\epsilon_{b o m}\right)$ and particle fluxes need to be determined. The quantity $\epsilon_{\text {bom }}$ depends on two essential parameters of the microplasma, namely the gas temperature $(T)$ and the electron density $\left(n_{e}\right)$. We used optical emission spectroscopy (OES) to obtain both of these plasma parameters. Due to the small length scales of a microplasma, diagnosing the parameters can be very difficult without perturbing the plasma. OES is an established, non-intrusive method which is wellsuited to measuring these parameters, despite the small length scales involved $[26,27]$. On determining these plasma parameters, $\epsilon_{\text {bom }}$ was calculated using a model 
that incorporates high-voltage collisional sheaths with a capacitively-coupled, radio-frequency (RF) discharge [28]. Furthermore, particle fluxes incident on the electrode surface were determined, permitting surface cleaning times to be estimated.

Section 2.1 describes the apparatus used to obtain the spectroscopic measurements from a microplasma in the ion microtrap device. Central to the method presented is that $T$ and $n_{e}$ are determined from measured spectral linewidths. Section 2.2 details several different broadening mechanisms which need to be accounted for. The methods used to determine the parameters $T$ and $n_{e}$ are described in section 2.3. Section 3 describes the method for calculating $\epsilon_{b o m}$ and surface adsorbate removal times. Data from both pure and mixed-gas microplasmas is presented in section 4 . The use of the technique for surface treatment of ion microtrap electrodes is considered in section 5 .

\section{Experiment and method}

\subsection{Experimental setup}

This work used ion microtrap devices of a monolithic, $3 \mathrm{D}$ electrode geometry, similar to that described in our earlier publication [20]. The devices are made by metallisation and micromachining of a $350 \mu \mathrm{m}$-thick $\mathrm{SiO}_{2}$-on-Si wafer [21]; the Au electrode pattern is laid down on the $\mathrm{SiO}_{2}$. Figure 1a illustrates the crosssectional format and material system of the electrode microstructure. Figure $1 \mathrm{~b}$ shows an example chip packaged in a ceramic leadless chip carrier. For this investigation, two architectures of microtrap were used. The first is a linear array of three trapping zones (figure 1c) [20], whereas the second has seven trapping zones and a spatially separated ion loading zone (figure 1d); hereafter these are referred to as type A and type B microtraps respectively.

A microtrap device was cleaned with a low-power, oxygen microwave plasma asher for 2 minutes, after which it was exposed to atmosphere for up to 1 day. Following this, it was contained under vacuum in a stainless steel chamber, similar to the form of that detailed in [22], where the ceramic carrier forms the electrical feedthrough for the vacuum (see figure 2 ). This procedure is almost identical to that used to package a device for ion trapping experiments. Hydrocarbon surface adsorbates accumulate on the $\mathrm{Au}$ electrodes during exposure to air (and also during vacuum bakeout when preparing a device for ion trapping) [10]. In contrast to [22], butyl rubber under pressure forms the vacuum seal between the stainless steel and ceramic chip carrier. This sealing method is sufficient for pumping down to pressures $P=$ $10^{-7}$ mbar yet it permits rapid and straightforward swapping of devices under test. The vacuum pumping and gas lines are so configured as to permit pumping of the chamber, as well as the introduction of gas and gas mixtures at controlled partial pressures.

A capacitively-coupled RF microplasma is created in the $340 \mu \mathrm{m}$-wide microtrap aperture by applying a voltage (of amplitude $130 \mathrm{~V} \leqslant U_{R F} \leqslant 230 \mathrm{~V}$ and frequency $\Omega_{R F} / 2 \pi=23 \mathrm{MHz}$ ) to the $\mathrm{RF}$ electrodes of the device (see figure 1a). The RF signal source is a National Instruments PXI 5404 with variable attenuator (Minicircuits ZX73-2500-S+) for amplitude control. This signal is amplified (Minicircuits ZHL$5 \mathrm{~W}-1$ ) and drives a tank circuit which includes the microtrap. A bi-directional coupler (Minicircuits ZFBDC20-61HP+) between the amplifier and the tank circuit samples the forward and reflected powers which, with the measured build-up factor of the tank circuit, are used to calculate $U_{R F}$ on the electrodes. The microplasma could be achieved with $\mathrm{He}$ and a He: $\mathrm{N}_{2}$ gas mixture where both gases were from sources of $99.999 \%$ purity. Testing with $\mathrm{N}_{2}$ alone was also possible, but this required $U_{R F}$ beyond the range of the specific test chips used in this study. To generate the microplasma, the DC electrodes of the device (see figure 1a) were grounded and the compensation electrodes were unconnected. It was observed that by leaving the Si bulk $\left(\rho=2.6 \times 10^{-3} \Omega \mathrm{cm}\right)$ electrically floating, a more homogeneous and stable microplasma was produced in the aperture.

The apparatus used to record the optical emission spectrum from the microplasma is illustrated in figure 2 ; this is similar to others reported [26,29-31]. A pair of achromatic lenses image the microplasma emission into a multi-mode fibre. The lenses and fibre are fixed with respect to each other, however this assembly can be translated in three dimensions which affords some spatial resolution. Another achromatic lens couples the imaged light from the fibre into to an imaging spectrometer (Horiba iHR550). The spectrum is detected by an electron-multiplying CCD camera (Andor Newton DU971N-FI), and the extent of the microplasma generated is monitored by a CMOS camera (Thorlabs DCC3240M).

At the operating parameters studied, microplasma emission was relatively weak. To record a spectral lineshape with sufficient signal-to-noise at a specific set of microplasma operating parameters, 10 sequential EMCCD exposures, each of $10 \mathrm{~s}$ duration, were averaged. Sequential acquisitions permitted the detection of any time-varying microplasma instability. Without a microplasma operating, the mean background spectrum was recorded using the same detection parameters. The lineshape with background subtracted was used for analysis and determination of the plasma parameters. 

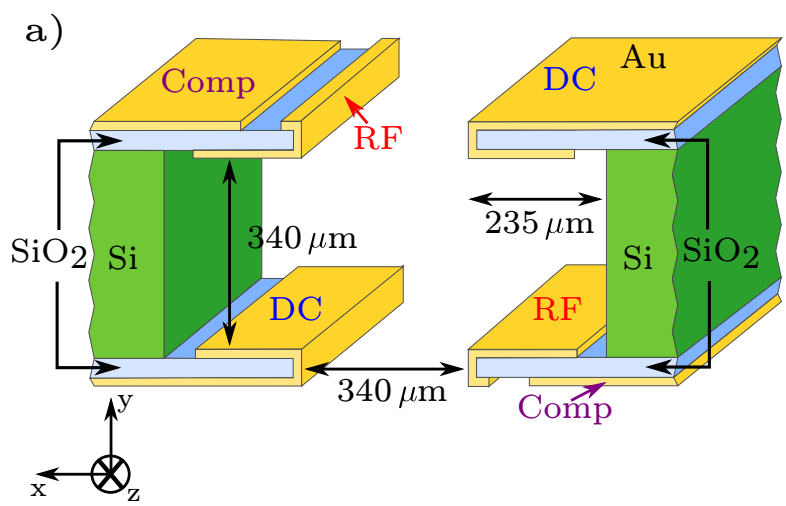

b)
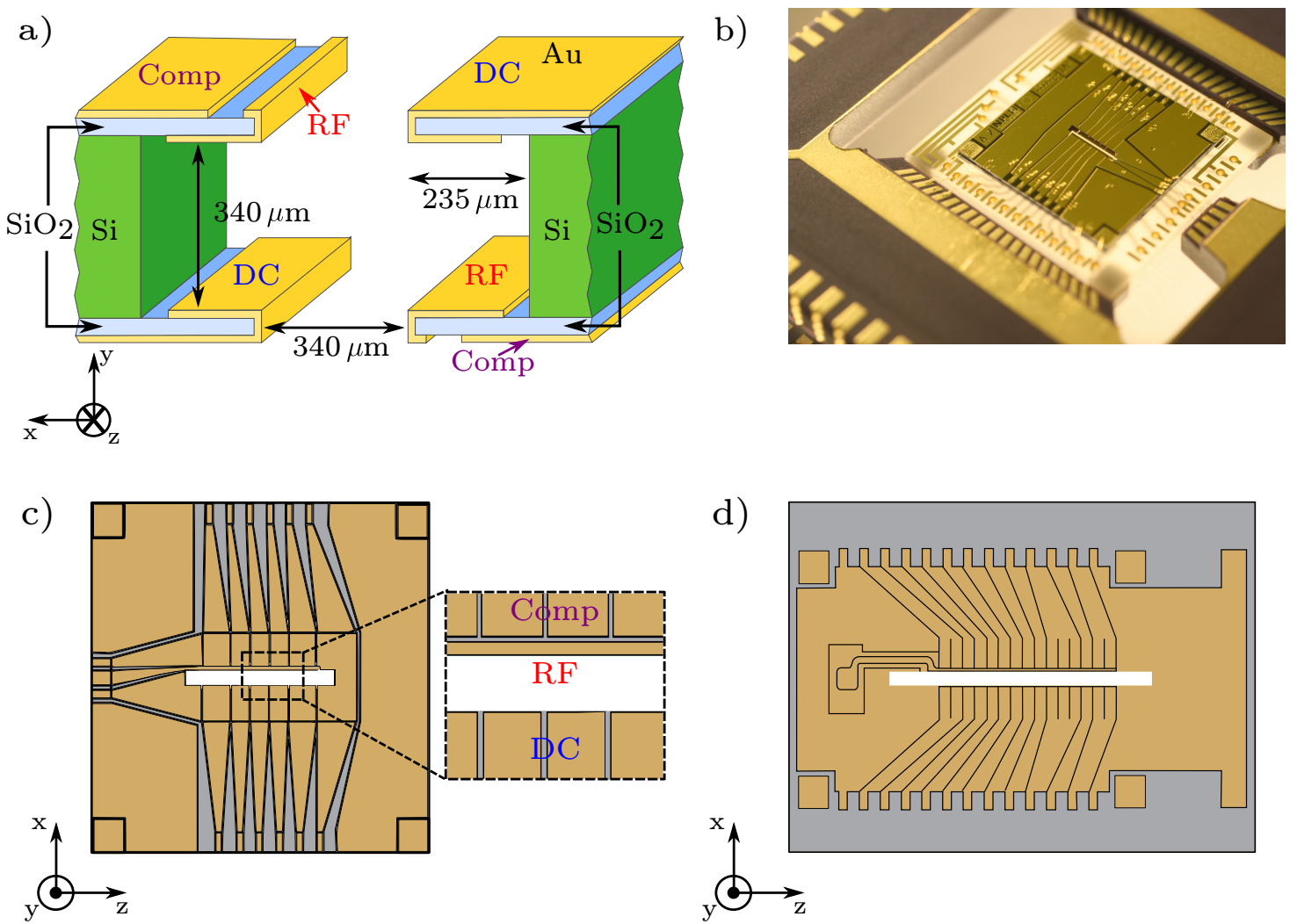

Figure 1. a) Schematic diagram showing the cross-sectional format of the trap chip aperture. This single-segment cut-out shows the material system of the ion microtrap. These features are identical for both type A and type B traps. Note that electrodes are on both front and back sides of the 3D micro-structure. b) Example of a type A microtrap in an electronic package. c) Front-side electrode layout of microtrap type A. The magnified inset shows the detail of the RF and DC electrodes around the trap aperture. Rotating the chip around the trap axis $(\mathrm{z})$ by $180^{\circ}$ would result in the same image from the back-side electrode layout. Chip dimensions are $8.4 \mathrm{~mm} \times 8.4 \mathrm{~mm}$ with a $2.6 \mathrm{~mm}$ long aperture. d) Front-side electrode layout of microtrap type B; as in c), the back-side layout is identical. Chip dimensions are $11.0 \mathrm{~mm} \times 8.4 \mathrm{~mm}$ with a $6.4 \mathrm{~mm}$ long aperture.

\subsection{Optical diagnostics}

To determine the gas temperature $(T)$ and the electron density $\left(n_{e}\right)$, the linewidths of two spectral features emitted by the microplasma were analysed; specifically these were the $\mathrm{He}$ I $667 \mathrm{~nm}$ line and the $\mathrm{H} \alpha \quad 656 \mathrm{~nm}$ Balmer Line [32]. In some microplasmas (where $n_{e} \leqslant 10^{12} \mathrm{~cm}^{-3}$ ), the Stark broadening of the He I $667 \mathrm{~nm}$ line is negligible [33]. However, in the experiments reported here $\left(10^{14} \mathrm{~cm}^{-3} \leqslant n_{e} \leqslant 10^{15} \mathrm{~cm}^{-3}\right)$, the Stark broadening of the He I $667 \mathrm{~nm}$ line must be taken into account [34]. Therefore, both measured spectral lines depend on $T$ and $n_{e}$. The $\mathrm{H} \alpha 656 \mathrm{~nm}$ line is particularly sensitive to $n_{e}$ and is well documented in the literature $[35,36]$. Despite no deliberate introduction of $\mathrm{H}$ into the system, the $\mathrm{H} \alpha$ line was evident and was attributed to dissociation of trace water vapour $[37,38]$ in the vacuum.

The spectral spread of optical emission from the microplasma consists of Lorentzian and Gaussian components which convolve to yield a Voigt profile. The linewidth of the Gaussian component, $\Delta \lambda_{G}$, is defined as

$$
\Delta \lambda_{G}=\left(\Delta \lambda_{I}^{2}+\Delta \lambda_{D}^{2}\right)^{1 / 2}
$$

where $\Delta \lambda_{I}$ and $\Delta \lambda_{D}$ are the contributions arising from instrument and Doppler broadening respectively. The spectrometer property $\Delta \lambda_{I}$, verified to be of Gaussian form, was measured using He-Ne laser radiation prior to each data run. A fit (using a Levenberg-Marqardt algorithm) to the resultant spectral lineshape gave values in the range $0.029(4) \mathrm{nm}$. The linewidth of the Lorentzian component, $\Delta \lambda_{L}$, is a combination of van der Waals, resonance and Stark broadening, which are quantified by linewidth contributions $\Delta \lambda_{V W}, \Delta \lambda_{R}$ and $\Delta \lambda_{S}$ respectively:

$$
\Delta \lambda_{L}=\Delta \lambda_{V W}+\Delta \lambda_{R}+\Delta \lambda_{S} .
$$

In the analysis that follows, equations quoted from the literature often contain particular quantities in non-SI 


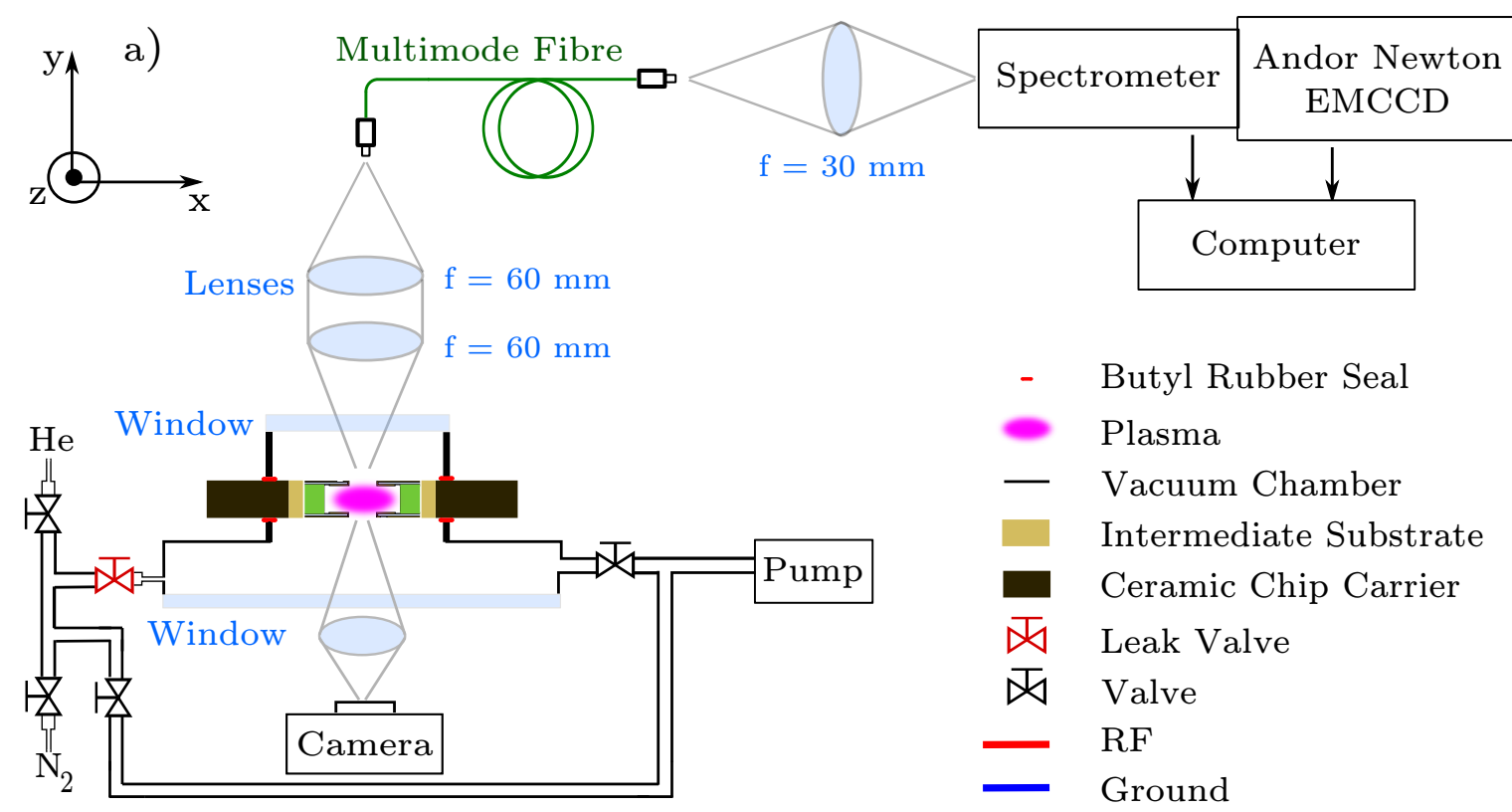

b)

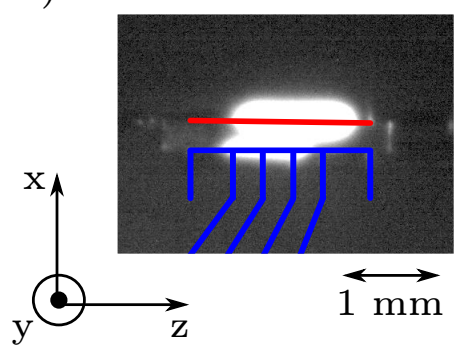

c)

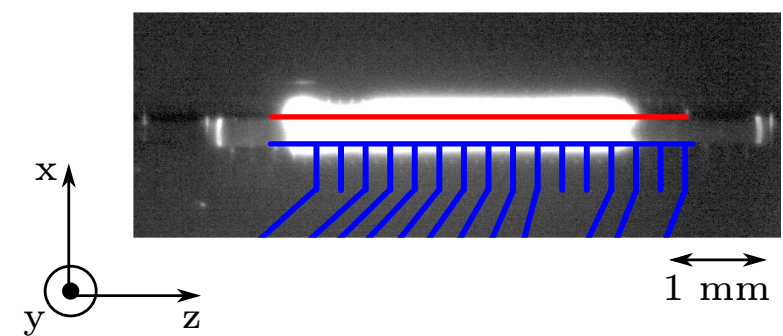

Figure 2. a) Experimental setup for optical emission spectroscopy. A pressure gauge (not shown) measures the vacuum and gas pressures in the chamber. The light emitted by the microplasma is imaged using a lens pair (with unit magnification) into a multimode fibre $(50 \mu \mathrm{m}$ core diameter, $0.22 \mathrm{NA}$ ). From the fibre, light is imaged (with magnification of 0.7 ) into a $0.55 \mathrm{~m}$ imaging spectrometer, where the detector array is an electron-multiplying CCD (pixel size of $16 \mu \mathrm{m}$ ). The spectrometer entrance slit width was set to $34 \mu \mathrm{m}$, and a 1200 lines/mm grating was used to achieve a measured resolution of 0.029(4) nm. Images of the microplasma were recorded by a CMOS camera at normal incidence to the trap aperture. b) Example of trap type A generating a He microplasma at $P=390 \mathrm{mbar}, \Omega_{R F} / 2 \pi=23 \mathrm{MHz}$ and $U_{R F}=154 \mathrm{~V}$. c) Example of trap type B generating a He microplasma at $P=400$ mbar, $\Omega_{R F} / 2 \pi=23 \mathrm{MHz}$ and $U_{R F}=160 \mathrm{~V}$. The electrode boundaries are overlaid on the images in b) and c). Exposure time was $93 \mathrm{~ms}$ for both images.

units for ease of use. In this section, wavelengths and linewidths are in units of nm. Other parameters with non-SI units are specified; otherwise SI units apply.

\subsubsection{Doppler broadening}

The velocity distribution of ions and atoms in the microplasma leads to a range of Doppler shifts associated with any spectral line. The resultant Doppler broadening contribution to the linewidth, $\Delta \lambda_{D}$, is well documented $[26,34,39,40]$, and can be written as

$$
\Delta \lambda_{D}=2.918 \times 10^{-20} \lambda_{0}\left(\frac{T}{M}\right)^{1 / 2}
$$

where $\lambda_{0}$ is the centre transition wavelength, $T$ is the temperature of the emitters and $M$ is atomic mass of the emitter. Here $T$ is assumed to be equal to the gas temperature in the microplasma due to the high collisional frequency between the emitters and neutral atoms.

\subsubsection{Resonance broadening}

Resonance (or self) broadening [41] occurs due to collisions with neighbouring identical species, where the transition giving rise to the spectral emission has an upper or lower energy level with an electric dipole coupling to the ground state [39]. The associated contribution to linewidth, $\Delta \lambda_{R}$, can be calculated using $[32,34,41]$ 


$$
\Delta \lambda_{R}=8.61 \times 10^{-28}\left(\frac{g_{L}}{g_{U}}\right)^{1 / 2} \lambda_{r} f_{r} \lambda_{0}^{2} n_{e m i t}
$$

where $g_{L(U)}$ is the statistical weight of the lower (upper) level of the transition, $\lambda_{r}$ and $f_{r}$ are respectively the resonance wavelength and the oscillator strength of the transition coupling to the ground state, and $n_{\text {emit }}$ is the density of emitter atoms in the ground state (in $\left.\mathrm{cm}^{-3}\right)$.

\subsubsection{Van der Waals broadening}

Van der Waals broadening results from the dipole of the excited emitter particle interacting with the induced dipole of the surrounding ground state atoms during a collisional process. The equations and relationships to quantify this broadening mechanism are detailed in [39] and reproduced here. The associated contribution to spectral linewidth, $\Delta \lambda_{V W}$, is given by

$$
\Delta \lambda_{V W}=8.18 \times 10^{-19} \lambda_{0}^{2}\left(R^{2} \alpha\right)^{2 / 5}\left(\frac{T}{\mu}\right)^{3 / 10} n_{g}
$$

where $R^{2}$ is a transition-dependent constant (in units of the Bohr radius $a_{0}$ ), $\alpha$ is the average polarisability (in $\mathrm{cm}^{3}$ ), $\mu$ is the emitter-perturber reduced mass (in amu) and $n_{g}$ is the neutral gas density (in $\left.\mathrm{cm}^{-3}\right)$. Here $R^{2}$ can be calculated as $R^{2}=R_{U}^{2}-R_{L}^{2}$, where $\mathrm{L}(\mathrm{U})$ is the lower (upper) energy level of the transition,

$$
R_{L(U)}^{2}=\frac{n_{L(U)}^{* 2}}{2}\left(5 n_{L(U)}^{* 2}+1-3 l_{L(U)}\left(l_{L(U)}+1\right)\right) .
$$

The orbital quantum number of the transition level is denoted by $l_{L(U)}$ and the square of the effective quantum number $n_{L(U)}^{* 2}$ is

$$
n_{L(U)}^{* 2}=\frac{I_{H}}{I_{I P}-E_{L(U)}}
$$

where $I_{H}$ is the ionisation potential of hydrogen, $I_{I P}$ is the ionisation potential of the emitter atoms (both in $\mathrm{cm}^{-1}$ ) and $E_{L(U)}$ is the energy of the lower or upper levels of interest (in $\mathrm{cm}^{-1}$ ). The average polarisability of the emitter can be estimated using

$$
\alpha=\frac{9}{2} a_{0}^{3}\left(\frac{3 I_{H}}{4 E_{e x}}\right)^{2}
$$

where the Bohr radius $a_{0}$ is in units of $\mathrm{cm}, E_{e x}$ (in $\mathrm{cm}^{-1}$ ) is the energy of the first excited level of the perturber particles.

\subsubsection{Stark broadening}

Due to the presence of charged perturbers in the microplasma such as ions and electrons, the Stark effect can broaden the spectral emission. The magnitude of the effect varies across different species. The Stark broadening contribution to the linewidth, $\Delta \lambda_{S}$, for $\mathrm{H}$ lines in an non-equilibrium discharge (as in this work), can be written as [42]

$$
\Delta \lambda_{S, H}=2.05 \times 10^{-11} n_{e}^{0.63}
$$

where $n_{e}$ is in $\mathrm{cm}^{-3}$. For He I lines, the analysis in [43] permits the Stark broadening of these lines to be calculated as

$$
\begin{aligned}
& \Delta \lambda_{S, H e}=2 \omega_{0}\left(\frac{n_{e}}{n_{e, 0}}\right) {\left[1+1.75 \alpha_{0}\left(\frac{n_{e}}{n_{e, 0}}\right)^{1 / 4} \times\right.} \\
&\left.\left(1-0.068 n_{e}^{1 / 6} T_{e}^{-1 / 2}\right)\right],
\end{aligned}
$$

where the electron impact half-width $2 \omega_{0}$ (in $\mathrm{nm}$ ) and the ion broadening parameter $\alpha_{0}$ can be found for a range of values of the reference electron density $n_{e, 0}$ (in $\mathrm{cm}^{-3}$ ) and $T_{e}[43,44]$.

\subsection{Lineshape fitting procedure}

This section describes how the plasma parameters $T, n_{e}$ (which are necessary for calculating $\epsilon_{b o m}$ ) were determined from spectral emission at a specific combination of $P$ and $U_{R F}$. A particular problem with fitting a Voigt function to a spectral feature is that the Gaussian and Lorentzian components can vary over a wide range, yet produce very similar lineshapes [26]. For example, the $\mathrm{H} \alpha 656 \mathrm{~nm}$ lineshape is a function of $T$ and $n_{e}$, but due to the low signal-to-noise ratio of this emission, multiple solutions are possible. Therefore a two-line Voigt function was fitted (using a LevenbergMarquardt algorithm) to a spectrum consisting of the $\mathrm{H} \alpha 656 \mathrm{~nm}$ and the He I $667 \mathrm{~nm}$ lines; the linewidths are dependent on $T$ and $n_{e}$, which were free parameters in the fit.

The function fitted to the two-line spectrum was

$$
f_{V, H, H e}=f_{V, H}+f_{V, H e}
$$

where the Voigt lineshape $f_{V, x}(x \in\{H, H e\})$ is given by $[26]$

$$
\begin{aligned}
& f_{V, x}=\frac{2 \ln 2 \Delta \lambda_{L, x}}{\pi^{3 / 2} \Delta \lambda_{G, x}^{2}} \times \\
& \int_{-\infty}^{+\infty} \frac{\exp \left(-t^{2}\right)}{\left(2(\ln 2)^{1 / 2} \frac{\left(\lambda-\lambda_{0}\right)}{\Delta \lambda_{G, x}}-t\right)^{2}+\left((\ln 2)^{1 / 2} \frac{\Delta \lambda_{L, x}}{\Delta \lambda_{G, x}}\right)^{2}} d t .
\end{aligned}
$$


The lineshapes $f_{V, H}$ and $f_{V, H e}$ have components $\Delta \lambda_{L}$ and $\Delta \lambda_{G}$ appropriate for the $\mathrm{H} \alpha$ and He I spectral lines. The constants associated with fitting a Voigt lineshape to these spectral features are listed in table 1.

Table 1. Constants, defined in the text, which are used in the fitting procedure outlined in section 2.3. $\mathrm{He}$ and $\mathrm{H} \alpha$ denote the He I $667 \mathrm{~nm}$ and $\mathrm{H} \alpha 656 \mathrm{~nm}$ transitions respectively. In lines 9 - 12, the subscripts $a$ and $b$ denote values corresponding to an electron temperature $T_{e}=20000 \mathrm{~K}$ and $40000 \mathrm{~K}(1.72 \mathrm{eV}$ and $3.45 \mathrm{eV}$ ) respectively, which covers the range in the experiments reported here. These $\omega_{0}$ and $\alpha_{0}$ are referenced at $n_{e, 0}=$ $10^{18} \mathrm{~cm}^{-3}$.

\begin{tabular}{llll}
\hline Transition & Symbol & Value & Reference \\
\hline $\mathrm{He}$ & $g_{L}$ & 1 & {$[41]$} \\
$\mathrm{He}$ & $g_{U}$ & 3 & {$[41]$} \\
$\mathrm{He}$ & $\lambda_{r}$ & $58.4334 \mathrm{~nm}$ & {$[41]$} \\
$\mathrm{He}$ & $f_{r}$ & 0.2762 & {$[41]$} \\
$\mathrm{He}$ & $I_{I P}$ & $198311 \mathrm{~cm}^{-1}$ & {$[41]$} \\
$\mathrm{He}$ & $E_{L}$ & $171135 \mathrm{~cm}^{-1}$ & {$[41]$} \\
$\mathrm{He}$ & $E_{U}$ & $186105 \mathrm{~cm}^{-1}$ & {$[41]$} \\
$\mathrm{He}$ & $E_{e x}$ & $171135 \mathrm{~cm}^{-1}$ & {$[41]$} \\
$\mathrm{He}$ & $\omega_{0 a}$ & $3.66 \mathrm{~nm}$ & {$[44]$} \\
$\mathrm{He}$ & $\omega_{0 b}$ & $3.29 \mathrm{~nm}$ & {$[44]$} \\
$\mathrm{He}$ & $\alpha_{0 a}$ & 0.52 & {$[44]$} \\
$\mathrm{He}$ & $\alpha_{0 b}$ & 0.56 & {$[44]$} \\
$\mathrm{He}, \mathrm{H} \alpha$ & $I_{H}$ & $109737 \mathrm{~cm}^{-1}$ & {$[39]$} \\
$\mathrm{H} \alpha$ & $E_{L}$ & $82259 \mathrm{~cm}^{-1}$ & {$[45]$} \\
$\mathrm{H} \alpha$ & $E_{U}$ & $97492 \mathrm{~cm}^{-1}$ & {$[45]$} \\
& & & \\
\hline
\end{tabular}

Since the $\mathrm{H} \alpha$ emission arises from a trace gas, $\Delta \lambda_{R}$ was neglected in that instance. Using the relations shown in equations (1) - (3), (5) and (9), $\Delta \lambda_{G, H}$ and $\Delta \lambda_{L, H}$ are described by

$$
\Delta \lambda_{G, H}=\left(\Delta \lambda_{I}^{2}+5.129 \times 10^{-13} \lambda_{0}^{2} T\right)^{1 / 2}
$$

and

$\Delta \lambda_{L, H}=\left(4.652 \times 10^{-11} \frac{P \lambda_{0}^{2}}{T^{7 / 10}}\right)+\left(2.05 \times 10^{-11} n_{e}^{0.63}\right)$,

where $n_{e}$ is in $\mathrm{cm}^{-3}$.

The He I $667 \mathrm{~nm}$ line depends on $\Delta \lambda_{I}, \Delta \lambda_{D}, \Delta \lambda_{R}$, $\Delta \lambda_{V W}$ and $\Delta \lambda_{S}$. From equations (3), (4), (5) and (10) the He line is also a function of $T$ and $n_{e}$. The Voigt function in equation (12) can then be re-written with $\Delta \lambda_{G, H e}$ and $\Delta \lambda_{L, H e}$ in the form

$$
\Delta \lambda_{G, H e}=\left(\Delta \lambda_{I}^{2}+1.282 \times 10^{-13} \lambda_{0}^{2} T\right)^{1 / 2}
$$

and

$$
\begin{aligned}
& \Delta \lambda_{L, H e}= \\
& \left(3.537 \times 10^{-11} \frac{P \lambda_{0}^{2}}{T^{7 / 10}}\right)+\left(5.811 \times 10^{-10} \frac{P_{e m i t} \lambda_{0}^{2}}{T}\right)+ \\
& \frac{2 \omega_{0} n_{e}}{10^{18}}\left(1+\alpha_{0} n_{e}^{1 / 4} 1.75 \times 10^{-4.5}\left(1-0.068 n_{e}^{1 / 6} T_{e}^{-1 / 2}\right)\right),
\end{aligned}
$$

where $P_{\text {emit }}$ is the partial pressure of the emitters and $n_{e}$ is in $\mathrm{cm}^{-3}$. The Stark broadening parameters $\omega_{0}$ and $\alpha_{0}$ are referenced at $n_{e, 0}=10^{18} \mathrm{~cm}^{-3}$ and the values used in this work can be found in table 1 . A linear interpolation between documented values was used in order to determine the parameters for a given $T_{e}$. Equation (16) depends on $T_{e}$, which in turn is a function of $T$. The method used to calculate $T_{e}$ for a given $T$ can be found in Appendix A.1. Since $T_{e}$ cannot be solved analytically, then for each iteration of the fitting routine, a new value for $T_{e}$ is calculated numerically (using a Newton-Raphson method). An example of a fit used to determine $n_{e}$ and $T$ is shown in figure 3. For ease of comparison to other published data, $n_{e}$ and $T$ can be applied to the relevant equations in section 2.2 to calculate broadening contributions $\Delta \lambda_{D}, \Delta \lambda_{V W}, \Delta \lambda_{S}$ and $\Delta \lambda_{R}$.

\section{Quantifying surface effects}

Once $T$ and $n_{e}$ have been measured, three plasma parameters must be calculated, to enable subsequent calculation of the principal parameters of interest. Appendix A describes the detail of how the electron temperature $T_{e}(T)$, the ion velocity $u_{s}\left(T, n_{e}\right)$ and density $n_{s}\left(T, n_{e}\right)$ at the sheath edge were calculated. From these three parameters, the average surface bombardment energies for ions can be calculated using models set out in [28] and [46]. Additionally, the flux of particles with sufficient energy to sputter surface adsorbates can be determined; in turn, this can be used to estimate times required to clean the microtrap electrode surfaces from adsorbates. The discharge studied in this work is a low-frequency, capacitively-coupled discharge at high pressure and with high-voltage, collisional sheaths. This specific regime permits some standard approximations in the calculations. In the equations presented in this section, all quantities are in SI units. However, specific values for energies are quoted in $\mathrm{eV}$ to be consistent with the literature on this topic.

\subsection{Calculating the ion bombardment energy}

In a collisional microplasma, the maximum sheath thickness $s_{m}$ is greater than the mean free path $\lambda_{i}$ of an ion [28]. As an ion is accelerated across the sheath, the 

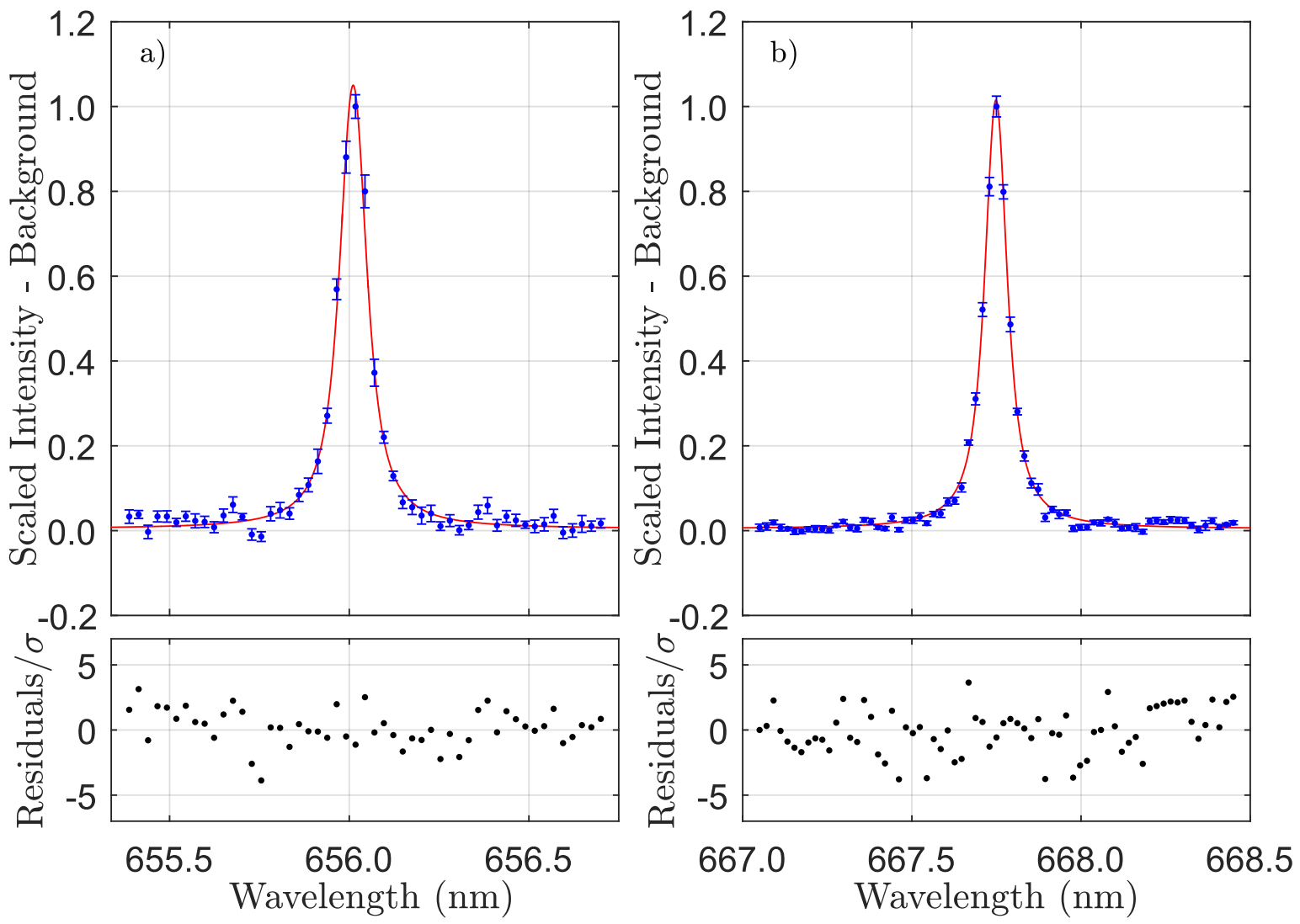

Figure 3. Examples of He I $667 \mathrm{~nm}$ and $\mathrm{H} \alpha 656 \mathrm{~nm}$ spectral line data observed at plasma parameters of $P=590 \mathrm{mbar}, \Omega_{R F} / 2 \pi=$ $23 \mathrm{MHz}$ and $U_{R F}=182 \mathrm{~V}$. From the fit to both lineshapes (see equations (11) - (16)), $T=323(12) \mathrm{K}$ and $n_{e}=5.8(7) \times 10^{14} \mathrm{~cm}^{-3}$, where the errors are $1 \sigma$ statistical from the fit only. a) $\mathrm{H} \alpha 656 \mathrm{~nm}$ line data (blue points) and Voigt lineshape fitted using equations (12), (13) and (14). Broadening contributions: $\Delta \lambda_{I}=0.0261 \mathrm{~nm}, \Delta \lambda_{D}=0.0085 \mathrm{~nm}, \Delta \lambda_{V W}=0.0207 \mathrm{~nm}, \Delta \lambda_{S}=0.0408 \mathrm{~nm}$. The studentised residuals are shown below the lineshape. b) He I $667 \mathrm{~nm}$ line data (blue points) and Voigt lineshape (red line) fitted using equations (12), (15) and (16). Broadening contributions: $\Delta \lambda_{I}=0.0261 \mathrm{~nm}, \Delta \lambda_{D}=0.0043 \mathrm{~nm}, \Delta \lambda_{V W}=0.0183 \mathrm{~nm}, \Delta \lambda_{R}=0.0473 \mathrm{~nm}$, $\Delta \lambda_{S}=0.0046 \mathrm{~nm}$. The studentised residuals are shown below the lineshape.

resulting collisions dissipate energy and lead to lower surface bombardment energies. Here $\lambda_{i}$ is calculated using the hard sphere model via [28]

$$
\lambda_{i}=\frac{k_{B} T}{\sigma P}
$$

where $k_{B}$ is the Boltzmann constant and $\sigma$ is the collisional cross-section for the particular particle interaction in question [28]. For a He-He collision $\sigma_{\mathrm{He}-\mathrm{He}}=1.36 \times 10^{-19} \mathrm{~m}^{2}[46] . \quad$ For a $\mathrm{He}^{+}-\mathrm{He}$ interaction we calculate the mean of the energydependent cross-section in the $1-10 \mathrm{eV}$ range to be $\sigma_{H e^{+}-H e}=3.10 \times 10^{-19} \mathrm{~m}^{2}[28,47]$.

The typical mean free path of an ion is in the submicron range. In addition, the time-varying sheath thickness is given by [28]

$$
s(t)=\left[1.43 \epsilon_{0}\left(\frac{2 e \lambda_{i}}{\pi M}\right)^{1 / 2} \frac{V_{s}(t)^{3 / 2}}{e n_{s} u_{s}}\right]^{2 / 5}
$$

where $e$ is the electronic charge, $M$ is the ion mass, $\epsilon_{0}$ is the free-space permittivity, and $V_{s}(t)$ is the timevarying sheath voltage.

Since the discharge is of low-frequency character, i.e. the RF drive frequency is much less than the ion plasma oscillation frequency, the ions respond to the instantaneous electric field. Furthermore, the electron energy relaxation time $(\sim 3 \mathrm{ps})$ is shorter than the $\mathrm{RF}$ period (43 ns) and as a result, the electron energy distribution is strongly modulated by the applied RF field. Therefore, the sheath can be approximated as a succession of DC sheaths at different points in the $\mathrm{RF}$ phase [27]. The sheath voltage $V_{s}$ for a lowfrequency plasma with high-voltage sheaths, i.e. where $e V_{s} \gg k_{B} T_{e}$, is [28]

$$
V_{s}= \begin{cases}U_{R F} \sin \left(\Omega_{R F} t\right), & 2 n \pi<\Omega_{R F} t<(2 n+1) \pi, \\ 0, & (2 n-1) \pi \leqslant \Omega_{R F} t \leqslant 2 n \pi .\end{cases}
$$

For the plasma regime in this work, the ion 
bombardment energy, and the flux energy distributions of the ions and neutrals discussed in section 3.2, can be found following the analysis of Hagelaar et. al. [46]. The time-varying mean ion bombardment energy $\gamma$ is given by

$$
\gamma=\frac{e E}{n_{g} \sigma_{H e^{+}-H e}} .
$$

Here, $n_{g}$ is the gas density, $\sigma_{H e^{+}-H e}$ refers to collisions in the sheath and the electric field $E$ in the sheath is $[28]$

$$
E=\left(\frac{3 e n_{s} u_{s}}{2 \epsilon_{0}\left(2 e \lambda_{i} / \pi M\right)^{1 / 2}}\right)^{2 / 3} s^{2 / 3},
$$

where $s$ has a time dependence according to equation (18). The time-averaged mean bombardment energy $\epsilon_{\text {bom }}=\frac{1}{t} \int_{0}^{t} \gamma d t$ is calculated using equations (18), (20) and (21). The sheath voltage is effective at accelerating the ions to the electrode over only half of the RF cycle (equation (19)), thus

$$
\epsilon_{\text {bom }}=\frac{e E_{0}}{\pi n_{g} \sigma} \int_{0}^{\pi}(\sin \theta)^{2 / 5} d \theta
$$

where $\theta=\Omega_{R F} t$ and $E_{0}$ is the electric field when $V_{s}=U_{R F}$.

\subsection{Estimating the adsorbate removal time}

As ions are accelerated in the sheath, collisions result in a flux of both neutral atoms (with timevarying energy distribution $\Gamma_{n}(\epsilon) d \epsilon$ ) and ions (with time-varying energy distribution $\Gamma_{i}(\epsilon) d \epsilon$ ) impacting upon the electrode surface. Approximate surface processing times can be calculated for both the ions and neutral atoms; this relies on knowing the timeaveraged particle flux incident on the electrode surface, $\Phi_{x}$ where $x \in\{i, n\}$.

Noting the current continuity condition [28] and subsequently that $\int_{0}^{\infty} \Gamma_{i}(\epsilon) d \epsilon=n_{s} u_{s}$, then at an instantaneous value of sheath voltage, the time-varying energy distribution of the ion flux at the surface is

$$
\Gamma_{i}(\epsilon) d \epsilon=\frac{n_{s} u_{s}}{\gamma} \exp \left(-\frac{\epsilon}{\gamma}\right) d \epsilon .
$$

To calculate $\Phi_{i}$ in an oscillating low-frequency sheath, the instantaneous ion flux must also be time-averaged over half of the RF cycle. After accounting for the $50 \%$ duty cycle of the sputtering process, $\Phi_{i}$ is given by

$$
\begin{aligned}
\Phi_{i}=\frac{1}{2 \pi} \int_{\epsilon_{t h}}^{\epsilon_{u p}} \int_{0}^{\pi} \frac{n_{s} u_{s}}{\gamma_{0}(\sin \theta)^{2 / 5}} \times \\
\exp \left(\frac{-\epsilon}{\gamma_{0}(\sin \theta)^{2 / 5}}\right) d \theta d \epsilon .
\end{aligned}
$$

Here, $\gamma_{0}$ is the instantaneous mean ion bombardment energy when $E=E_{0}$, and $\epsilon_{t h}=12.3 \mathrm{eV}$ is the sputtering threshold (see section 4). $\epsilon_{u p}$ is the absolute upper limit of the ion energy (i.e. no collisions when crossing the sheath at maximum potential), which is given by

$$
\epsilon_{u p}=\frac{M}{2}\left(\frac{2 s_{m} e E_{0}}{M}+u_{s}^{2}\right) .
$$

Typically, $330 \mathrm{eV} \leqslant \epsilon_{u p} \leqslant 500 \mathrm{eV}$.

The bounds of the neutral flux energy distribution $\Gamma_{n}(\epsilon) d \epsilon$ for an instantaneous sheath voltage are given in [46]. The upper and lower limits respectively are given by

$$
\Gamma_{n}(\epsilon) d \epsilon<\frac{\sigma_{H e^{+}-H e}}{\sigma_{H e-H e}}\left(1+\frac{2 \gamma}{\epsilon}+\frac{2 \gamma^{2}}{\epsilon^{2}}\right) \Gamma_{i}(\epsilon) d \epsilon
$$

and

$$
\Gamma_{n}(\epsilon) d \epsilon>\frac{\sigma_{H e^{+}-H e}}{\sigma_{H e-H e}} \Gamma_{i}(\epsilon) d \epsilon .
$$

$\Phi_{n}$ is calculated in a similar fashion to $\Phi_{i}$ as above, at both limits of $\Gamma_{n}(\epsilon) d \epsilon$. From these two limits, a mean value of $\Phi_{n}$ is calculated. In doing so, this requires calculation of $\sigma_{H e^{+}-H e}[28,47]$ in the range $\epsilon_{t h} \leqslant \epsilon \leqslant \epsilon_{u p}$; we use a mean value (weighted with respect to $\Gamma_{n}(\epsilon) d \epsilon$ ) of $\sigma_{H e^{+}-H e}=2.1 \times 10^{-19} \mathrm{~m}^{2}$.

The removal of an adsorbed hydrocarbon layer is described by the rate equation [48]

$$
\frac{d N_{a}}{d t}=-\Phi_{x} \sigma_{a} N_{a}(t)
$$

where $N_{a}$ is the adsorbate surface density and $\sigma_{a}$ is the sputtering cross-section calculated following the approach of $[48,49]$. Note that a limitation of this approach is that it does not take into account redeposition of the sputtered material through processes such as back-diffusion and back-scattering $[50,51]$.

Solving equation (28) gives $N_{a}=N_{0} \exp \left(-t / \tau_{a}\right)$, where $N_{0}$ is the initial adsorbate surface density and $\tau_{a}=\left(\Phi_{x} \sigma_{a}\right)^{-1}$ is the time constant for the process. The time taken to reach a target surface density for the adsorbate, $N_{t}$, is then given by

$$
t_{\text {clean }}=\left(\ln \frac{N_{0}}{N_{t}}\right) \tau_{a} .
$$

An estimate of $N_{a}(t=0)=N_{0}$ for a hydrocarbon adsorbate was made on the basis that the average $\mathrm{H}$ $\mathrm{C}$ bond length is $110 \mathrm{pm}$ [52]; assuming a grid of these bonds in two monolayers, we estimate $N_{0}=$ $4.1 \times 10^{19} \mathrm{~m}^{-2}$. In reality, the hydrocarbon density will depend on the exact structure of the hydrocarbons themselves and the adsorption sites that exist on the electrode surface. 


\section{Results and discussion}

In the remainder of this paper we quote values for energies, electron density and pressure in $\mathrm{eV}, \mathrm{cm}^{-3}$ and mbar for consistency with the literature on this topic.

It is advantageous to maximise the microplasma coverage of electrodes, to minimise redeposition of sputtered particles [51] onto trapping electrode surfaces. Figures 2b,c present two example images to illustrate the coverage that was attained. Quantitative data on the electrode coverage for a single trap chip of each type is presented in figure 4 . This shows that adequate coverage of the trapping zones can be achieved for trap type B at 330 mbar $\leqslant P \leqslant 440$ mbar. In trap type A the microplasma covers both sides of the aperture less symmetrically than in type B, resulting in incomplete trapping zone coverage. However at data points corresponding to $P=\{390,450,490\}$ mbar coverage is almost complete. Improved coverage would result from a higher $U_{R F}$, but this was not possible due to limitations of the particular test device used here.

Four microtrap chips, two of each device type, were used to make repeated spectroscopic measurements in a pure He microplasma. Figure 5a shows the voltage amplitudes $U_{R F}$ at which the plasma spectroscopic measurements were recorded. These amplitudes do not correspond to the threshold for plasma breakdown, but instead to the conditions where the microplasma had the greatest coverage and homogeneity over the extent of the device aperture. The spectroscopy measurements were recorded over a pressure range of 350 - 910 mbar for trap type A and a range of 290 - 490 mbar for trap type B. Two primary factors limited the ranges of $U_{R F}$ and pressure. First, the microplasma can become delocalised; we observed light emitted outside the aperture and along the tracks on the surface of the device. Secondly, there was also the risk of generating a microplasma at the wirebonds that make the electrical connection from the ceramic chip carrier to the trap. In both cases, a plasma constriction could sputter the electroplated $\mathrm{Au}$ and result in electrical shorts. With the normal microplasma operating conditions established, the plasma was confined to the microtrap aperture when struck.

The gas temperature $T$ and electron density $n_{e}$ were determined using the methods in section 2 and the results can be seen in figure 5b,c. For a microplasma maximally distributed in the trap aperture, $T$ shows a positive correlation with pressure, ranging from around room temperature up to as much as $\sim 460 \mathrm{~K}$. This is to be expected from pressure alone, however note that for some data sets, the increase in $U_{R F}$ accompanying the pressure increase (see figure 5a) is also expected to raise $T[33,40,42,53]$. Typical values of $T$ for microplasmas under similar conditions have been reported in the range $300 \mathrm{~K} \lesssim T \lesssim 600 \mathrm{~K}[32,33,54]$. A low gas temperature in the microplasma is beneficial since it decreases the possibility of thermal damage to the microtrap. The error bars in figure 5 comprise uncertainties due to the statistics of the fit $(1 \sigma)$, the Stark and instrument broadening components, and in some instances a small but noticeable deviation from the ideal Voigt lineshape.

It has been pointed out in other work [55] that semiclassical theory calculations of neutral atom Starkbroadened linewidths agree with experiment to within $\pm 20 \%$. From analysis of the fitting routine, we estimate that this contributes additional uncertainties of only $\pm 2 \%$ to $T$ and $\pm 1 \%$ to $n_{e}$. We estimate that the uncertainty in the instrument broadening contributes uncertainties of $\pm 1 \%$ in $T$ and $\pm 2 \%$ in $n_{e}$. For a few data points, the results of the fitting routine appear to suggest values of $T$ below room temperature. However, in those instances we observe that the wings of the He I lineshape deviate by a small amount from the ideal Voigt profile (the cause of this is not known). In such cases, the fit overestimates the Lorentzian component and thus underestimates $T$. From further analysis, we estimate that the associated systematic errors in $T$ and $n_{e}$ are $+45 \mathrm{~K}$ and $+4 \times 10^{13} \mathrm{~cm}^{-3}$ for those particular points. In those cases, these errors are added to the upper error bounds of both quantities. For those points, $T$ then extends above room temperature.

Micro-scale plasmas operate at a much higher pressure than those on a larger scale. As a result, they can also obtain higher densities of charged particles. The results for the measured $n_{e}$ are presented in figure 5c. A weighted, linear, least-squares fit to the measured $n_{e}$ showed a weak positive correlation with increasing pressure and voltage over the ranges tested. This observed trend is consistent with literature $[53$, 56].

Using the data in figures $5 \mathrm{~b}, \mathrm{c}$, the time-averaged mean bombardment energies $\epsilon_{\text {bom }}$ were calculated and are displayed in figure $5 \mathrm{~d}$. The results show that within the voltage and pressure ranges tested, the mean ion bombardment energy is $0.3 \mathrm{eV} \leqslant \epsilon_{\text {bom }} \leqslant 2.1 \mathrm{eV}$ (this is the range covered by the data and associated error bar extremes). These results are consistent with $\epsilon_{\text {bom }}$ calculated using numerical simulations of He microplasmas operating in a regime similar to that reported here. For example, simulations of microplasmas in a device with a $200 \mu \mathrm{m}$ electrode spacing, operating at $200 \mathrm{~V} \leqslant U_{R F} \leqslant 400 \mathrm{~V}, \Omega_{R F} / 2 \pi=$ $13.56 \mathrm{MHz}$ and $P=1000$ mbar, have shown mean bombardment energies that ranged from $1 \mathrm{eV} \leqslant$ $\epsilon_{\text {bom }} \leqslant 3 \mathrm{eV}[27]$.

During the proposed microplasma processing procedure, it is essential that while contaminants 

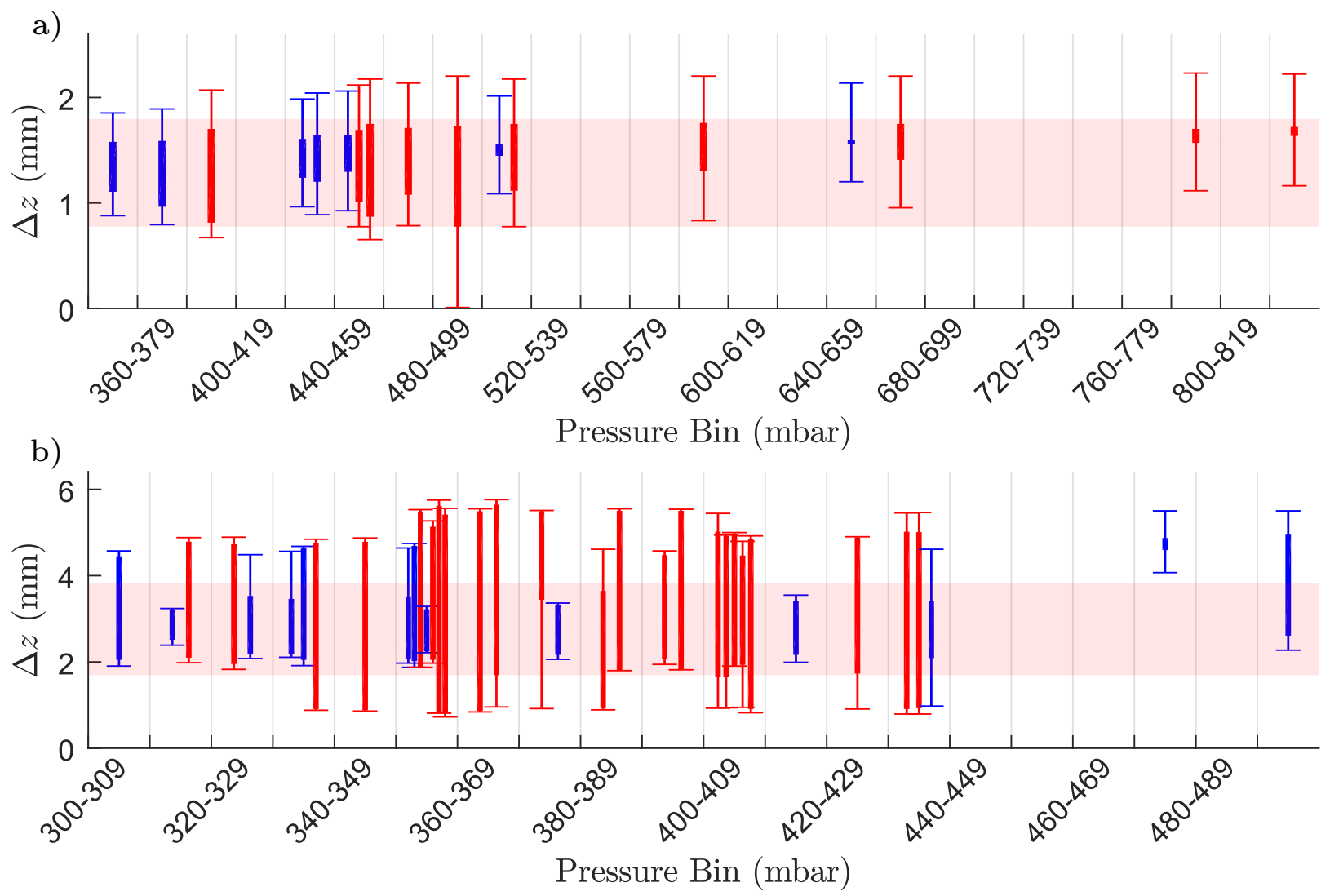

Figure 4. Example of aperture coverage in the axial direction $(\Delta z)$ attained for a) trap type A and b) trap type B, using a He microplasma at $\Omega / 2 \pi=23 \mathrm{MHz}$. For ease of displaying multiple data points at identical pressures, the data is binned into consecutive pressure ranges (20 mbar in type A and 10 mbar in type B). For each data point, the error bars denote the plasma extremes and the boxed region indicates where the plasma symmetrically covers both sides of the aperture in the axial direction. The shaded region in each figure corresponds to the trapping zones of each device, which are the principal electrodes requiring surface processing. a) Trap type A aperture coverage data taken at $U_{R F} \leqslant 150 \mathrm{~V}$ is in blue and $U_{R F}>150 \mathrm{~V}$ is in red. b) Trap type B aperture coverage data taken at $U_{R F} \leqslant 190 \mathrm{~V}$ is in blue and $U_{R F}>190 \mathrm{~V}$ is in red.

get removed, there is little or no damage to the actual electrode surface. The energy threshold for sputtering of amorphous hydrocarbons by He, $\epsilon_{t h}$, can be calculated using the equation [57]

$$
\epsilon_{t h}=\left[7.0\left(\frac{M_{C}}{M_{H e}}\right)^{-0.54}+0.15\left(\frac{M_{C}}{M_{H e}}\right)^{1.12}\right] \epsilon_{s b}
$$

where $M_{H e}$ and $M_{C}$ are the masses of $\mathrm{He}$ and $\mathrm{C}$ atoms respectively, and $\epsilon_{s b}=2.8 \mathrm{eV}$ is the surface binding energy for amorphous hydrocarbons [58]. From equation (30), $\epsilon_{t h}$ was calculated to be $12.3 \mathrm{eV}$; figure $5 \mathrm{~d}$ demonstrates that the microtrap devices operate in a regime where $\epsilon_{\text {bom }}$ is well below this threshold, and indeed substantially below the $57 \mathrm{eV}$ threshold for He sputtering $\mathrm{Au}$ [23]. Consequently, the processing procedure for removal of adsorbed hydrocarbon contaminants will be reliant on the upper tail of the energy distribution.

The estimated sputtering time constants, $\tau_{a}=$ $\left(\Phi_{x} \sigma_{a}\right)^{-1}$, for hydrocarbon surface adsorbates are presented in figure 6. Estimates for processing times $t_{\text {clean }}$, to achieve an arbitrary target adsorbate density $N_{t}$ of $1 \mathrm{~mm}^{-2}$, were calculated using equation (29) and a calculated value for $\sigma_{a}$. Up to $500 \mathrm{eV}, \sigma_{a}$ is only weakly dependent on projectile energy, so we approximate it with the mean value in that range, $\sigma_{a}=1.52 \times 10^{-20} \mathrm{~m}^{2}[48,49]$. The auxiliary axis of figure 6 also shows the data scaled to $t_{\text {clean }}$ for $N_{t}=1 \mathrm{~mm}^{-2}$. The estimated surface processing times varied upwards from $\sim 60 \mathrm{~s}$ over the $P$ and $U_{R F}$ ranges tested. Equation (29) shows that to reduce the adsorbate density by an extra factor of 100 requires an increase in $t_{\text {clean }}$ of only $4.6 \tau_{a}$. Figure 6 indicates that the flux of neutral atoms can have a substantial effect on the electrode surface that is comparable, if not greater, than the effect of the ions themselves. Other simulations and experiments have shown that fast neutrals can significantly contribute to the sputtering effect on the electrode surface [59-62]. For instance, in an $\mathrm{O}_{2}$ plasma sputtering a $\mathrm{Cr}$ surface at $P=0.01 \mathrm{mbar}$, $30 \%$ of the total rate of sputtering was attributed to 

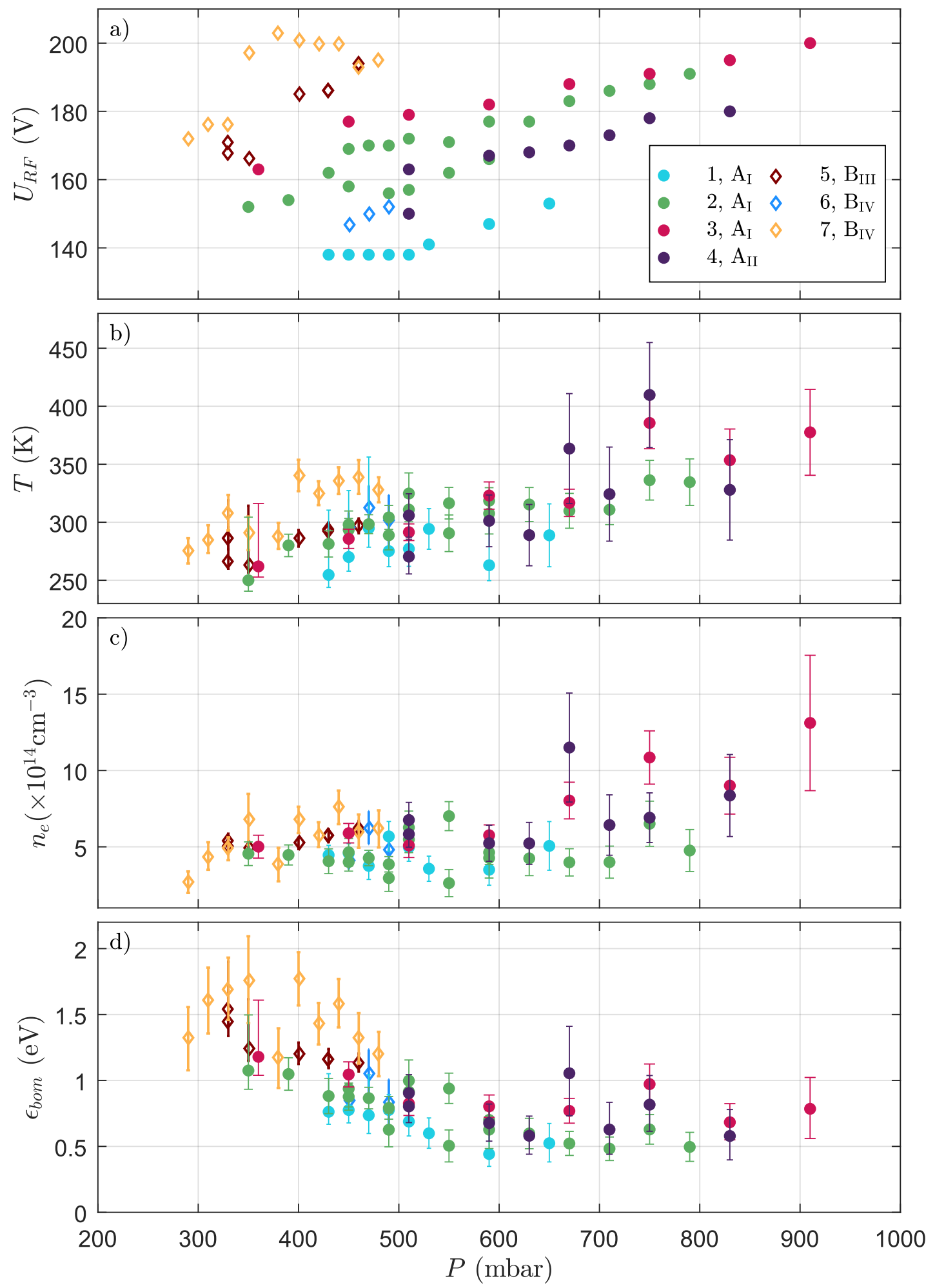

Figure 5. Measurements to determine the average ion bombardment energy with He at $\Omega_{R F} / 2 \pi=23$ MHz. Four individual devices were used (denoted by subscripts I - IV in the legend). a) Operating $U_{R F}$ values for a microplasma confined to the aperture with maximum coverage, for trials 1-4 in trap type A (circles) and trials 5-7 in trap type B (diamonds). b) $T$, and c) $n_{e}$, as measured from Voigt lineshapes fitted to the He I $667 \mathrm{~nm}$ and $\mathrm{H} \alpha 656 \mathrm{~nm}$ lines, using the $U_{R F}$ from (a) and the method in section 2.3 . d) $\epsilon_{b o m}$ calculated from measured results in (a-c) via time-averaging of equation (22). The uncertainties in $\epsilon_{b o m}$ values are propagated from those in $T$ and $n_{e}$, which are described in the text. The legend shown in a) applies to all plots. Between trials with the same device, removal from vacuum and subsequent pre-trial preparation could contribute to the spread of pressure-voltage characteristics. Minor defects (e.g. surface quality and wirebonding) in devices of the same type will also contribute to differences in these characteristics. 


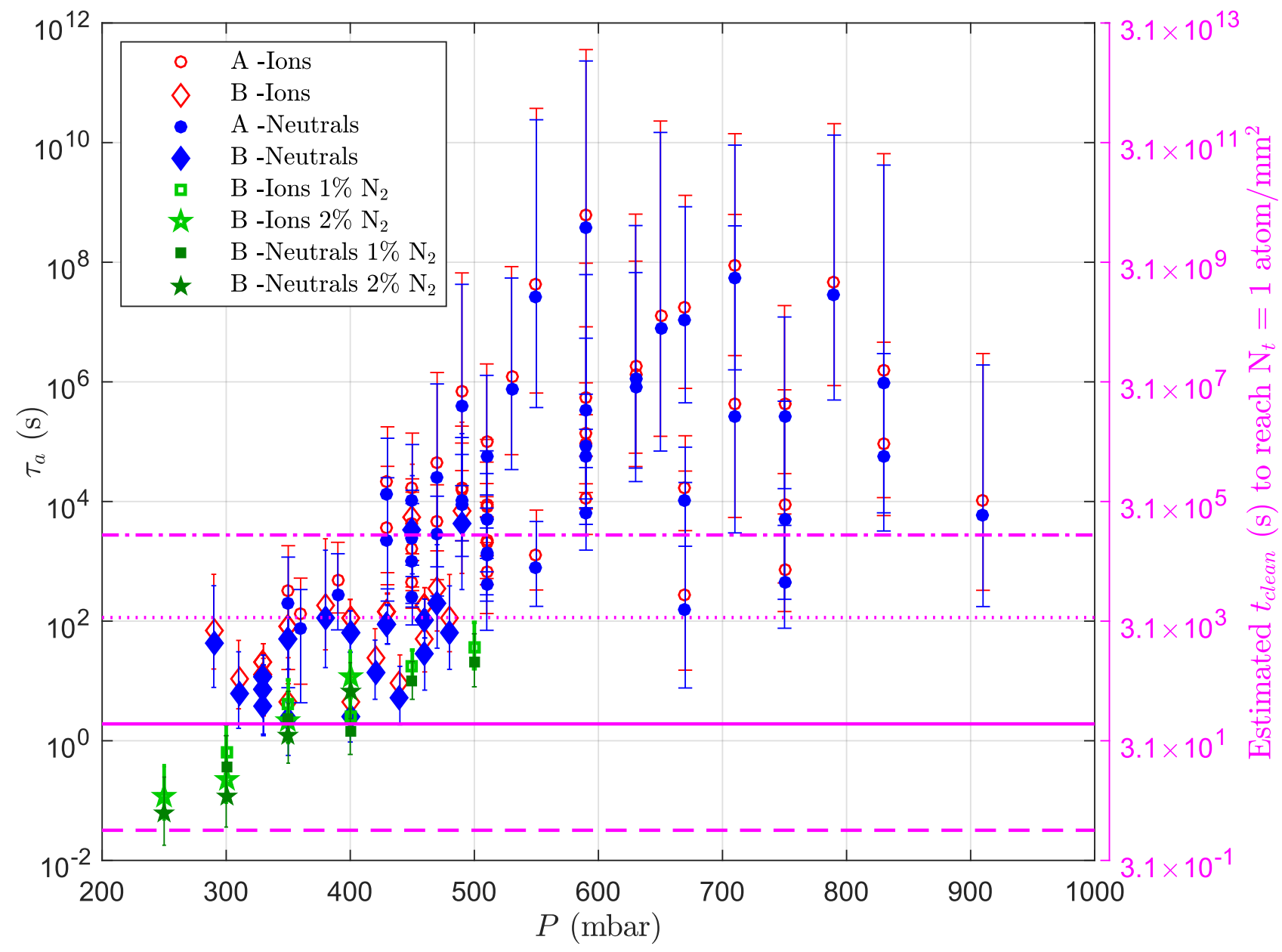

Figure 6. Estimated time constants $\tau_{a}$ for removal of hydrocarbon surface adsorbates on the microtrap electrodes. The estimates are based on fluxes calculated from measured plasma parameters. The $\tau_{a}$ due to ion flux are in open symbols, whereas those due to neutrals are in filled symbols. The latter are based on the mean of the upper and lower limits of the neutral flux. Error bars are propagated from the uncertainties in the spectroscopic measurements. The data is shown for He using trap types A and B (circles and diamonds respectively) and for trap type B using He with $1 \% \mathrm{~N}_{2}$ and $2 \% \mathrm{~N}_{2}$ (squares and stars respectively). The separated axis on the right of the graph shows the estimated time required to remove two monolayers of hydrocarbon adsorbates and reach a target adsorbate surface density of $N_{t}=1 \mathrm{atom} / \mathrm{mm}^{2}$. The coloured horizontal lines indicate surface cleaning times of $1 \mathrm{~s}$ (dashed), 1 minute (solid), 1 hour (dotted) and 1 day (dashed and dotted).

neutrals [61]. As the pressure increases and the ion undergoes more collisions in the sheath, the relative proportion of fast neutrals generated is expected to increase.

For trap type B, since the microplasma approaches a homogeneous spread at higher voltages, the ions are more energetic and the estimated surface processing time is less than that for trap type A. For a microplasma that is well distributed in the aperture of trap type A, the optimal processing parameters are likely to be around $P=360 \mathrm{mbar}$ and $U_{R F}=160 \mathrm{~V}$ for around 40 minutes. For trap type B, the optimum condition would be to operate the microplasma at $P=$ 350 mbar and $U_{R F}=200 \mathrm{~V}$, with $t_{\text {clean }}=60 \mathrm{~s}$. However, it should be noted that the model presented here is merely an approximation, since it does not account for the exact nature of the transportation of sputtered impurities. This same approximation was made in other work modelling microdischarges in coplanar geometries [60]. Sputtered contaminants could become negatively charged and confined within the plasma. Alternatively, after several collisions the sputtered particles can leave the plasma and diffuse in all directions to insignificant surfaces [51]. Finally, sputtered material may be redeposited on the electrode surfaces via back-diffusion or back-scattering processes [51]; in that case a greater $t_{\text {clean }}$ would be required.

The effect of the microplasma on the electrode material itself can be estimated by considering the time constant for removal of gold surface layers, $\tau_{A u}=$ $\left(\Phi_{H e, \max } \sigma_{H e-A u}\right)^{-1}$. Here, $\Phi_{H e, \max }$ is the maximum neutral He flux density in the range $\epsilon>\epsilon_{t h}=57 \mathrm{eV}$ (the Au sputtering threshold), and $\sigma_{H e-A u}=1.42 \times$ $10^{-20} \mathrm{~m}^{2}$ is the calculated sputtering cross-section for 
He with $\mathrm{Au}[48,49]$. In similar fashion to before, we calculate a weighted mean of $\sigma_{\mathrm{He}^{+}-\mathrm{He}}=1.2 \times$ $10^{-19} \mathrm{~m}^{2}$ for energies greater than $\epsilon_{t h}=57 \mathrm{eV}$. For the optimum processing parameters above, $\Phi_{H e, \max } \simeq$ $9.2 \times 10^{4} \mathrm{~m}^{-2} \mathrm{~s}^{-1}$ (trap type A) and $\Phi_{H e, \max } \simeq 7.1 \times$ $10^{10} \mathrm{~m}^{-2} \mathrm{~s}^{-1}$ (trap type B). Thus for the microplasmas observed in this work, we estimate $\tau_{A u, A} \simeq 7.8 \times 10^{14} \mathrm{~s}$ and $\tau_{A u, B} \simeq 1 \times 10^{9} \mathrm{~s}$ (where subscripts $\mathrm{A}$ and $\mathrm{B}$ denote the trap type), which are orders of magnitude greater than $\tau_{a}$. These values of $\tau_{A u}$ show that sputtering of $\mathrm{Au}$ is expected to be negligible. To avoid electrical breakdown, it is important that $\mathrm{Au}$ is not sputtered and redeposited on the insulating $\mathrm{SiO}_{2}$ surface (see figure 1a).

To verify that the electrodes were undamaged by the generation of the microplasma, images of internal $\mathrm{Au}$ surfaces in a type $\mathrm{B}$ ion trap were taken before and after an appropriate surface processing procedure (duration of 15 minutes, which for that particular data point was $\sim 27 \tau_{a}$ ). Images were recorded at five axial $(z)$ positions along the full extent of both an RF electrode, and also a DC electrode array. At each $z$ position, three images were recorded along the $x$ direction because the oblique viewing angle lacked sufficient depth of focus. In all positions but one, no difference was observed between the 'before' and 'after' images (see figure 7a,b). The end of the RF electrode was the one exception (see figure $7 \mathrm{c}, \mathrm{d}$ ) where a slight tarnishing near the corner tip was apparent. This effect is likely due to the localised high field emission which is a consequence of the electrode geometry. Since the effect is at a spatial location that is far removed from trapping zones it is considered to be negligible.

In addition to trials with pure $\mathrm{He}$, gas mixtures of $\mathrm{He}$ and $\mathrm{N}_{2}$ were tested. With $\mathrm{N}_{2}$ it is possible to chemically sputter the hydrocarbon contaminants from the electrode surface, as opposed to the physical sputtering by He. The particular test devices used in this study were limited in applied $U_{R F}$, due to plasma formation around wirebond connections outside of the device aperture. Since $\mathrm{N}_{2}$ has a higher breakdown voltage than $\mathrm{He}[63,64]$, this limitation restricted the concentrations of $\mathrm{N}_{2}$ in He to between $1 \%$ and $2 \%$. The results for a single data set using a type B trap can be seen in figure 8 . Further testing with the same trap has shown these results to be repeatable. The data for the He: $\mathrm{N}_{2}$ mixtures shows the bounds of $\epsilon_{\text {bom }}$ in the range $1.2 \mathrm{eV} \leqslant \epsilon_{\text {bom }}(\mathrm{He}) \leqslant 4.1 \mathrm{eV}$; these energies are approximately double those evident in the pure $\mathrm{He}$ microplasmas. From $P=400$ 500 mbar, the $U_{R F}$ limit of the device under test was less than the amplitude required to achieve a microplasma extending across the full length of the device aperture. Figure $8 \mathrm{e}$ shows that, with this limitation, microplasma coverage of the trapping zones

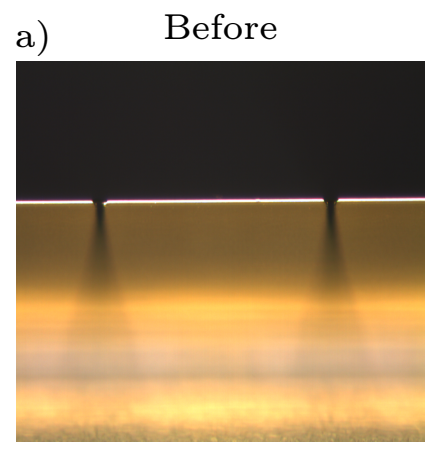

b) After

c)
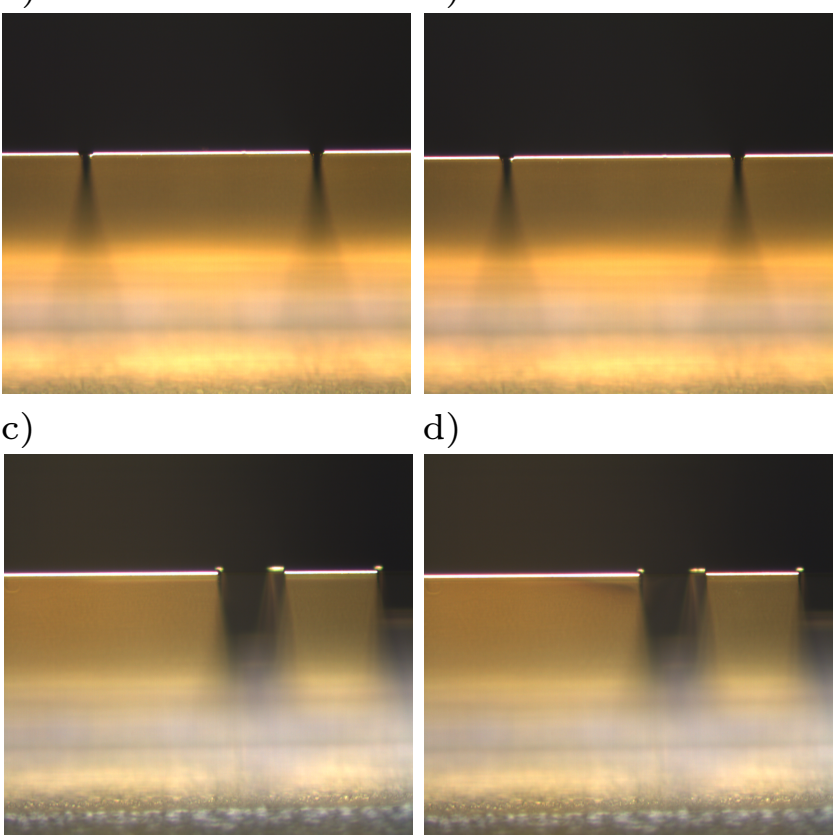

d)

Figure 7. Sample pictures of electrode internal surfaces before and after microplasma processing with $\mathrm{He}$ at $P=360$ mbar, $\Omega_{R F} / 2 \pi=23 \mathrm{MHz}$ and $U_{R F}=160 \mathrm{~V}$ for 15 minutes $\left(\sim 27 \tau_{a}\right.$, where $\tau_{a}=33 \mathrm{~s}$ for these operating parameters). Centre of DC electrode array a) before and b) after microplasma processing. Similarly, one end of an RF electrode c) before and d) after processing. These images were recorded with the electrode's edge nearest the aperture in focus.

was almost complete at $P=\{350,400\}$ mbar with $1 \% \mathrm{~N}_{2}$. To calculate $\epsilon_{\text {bom }}$ associated with the $\mathrm{He}$ ions the partial pressure of $\mathrm{He}: \mathrm{N}_{2}$ gas mixture was taken into account when calculating the sheath thickness $s$ in equation (18). The estimated $\tau_{a}$ for the He: $\mathrm{N}_{2}$ microplasmas are presented alongside the values for pure He microplasmas in figure 6. Similar to before, this also shows the estimated $t_{\text {clean }}$ to achieve $N_{t}=1 \mathrm{~mm}^{-2}$. This He: $\mathrm{N}_{2}$ data shows lower $\tau_{a}$ than for pure $\mathrm{He}$ at the lowest pressures, which is due to achieving higher values of $\epsilon_{\text {bom }}$. Estimated cleaning times are in the range $2 \mathrm{~s} \leqslant t_{\text {clean }} \leqslant 650 \mathrm{~s}$. These results suggest that a He: $\mathrm{N}_{2}$ gas mixture could reduce surface processing times, in comparison to pure He. A more stringent comparison is to consider only the data where maximum coverage of the trapping zones was achieved. In that case, with $1 \% \mathrm{~N}_{2}$ in $\mathrm{He}$ at $P=350$ mbar and 400 mbar, $t_{\text {clean }}=74 \mathrm{~s}$ and $43 \mathrm{~s}$ respectively. This is comparable to $t_{\text {clean }}=60 \mathrm{~s}$ at the optimum conditions for pure He as stated earlier.

\section{Conclusions and outlook}

We demonstrated a capacitively-coupled, RF microplasma inside the 3D electrode structure of an ion 

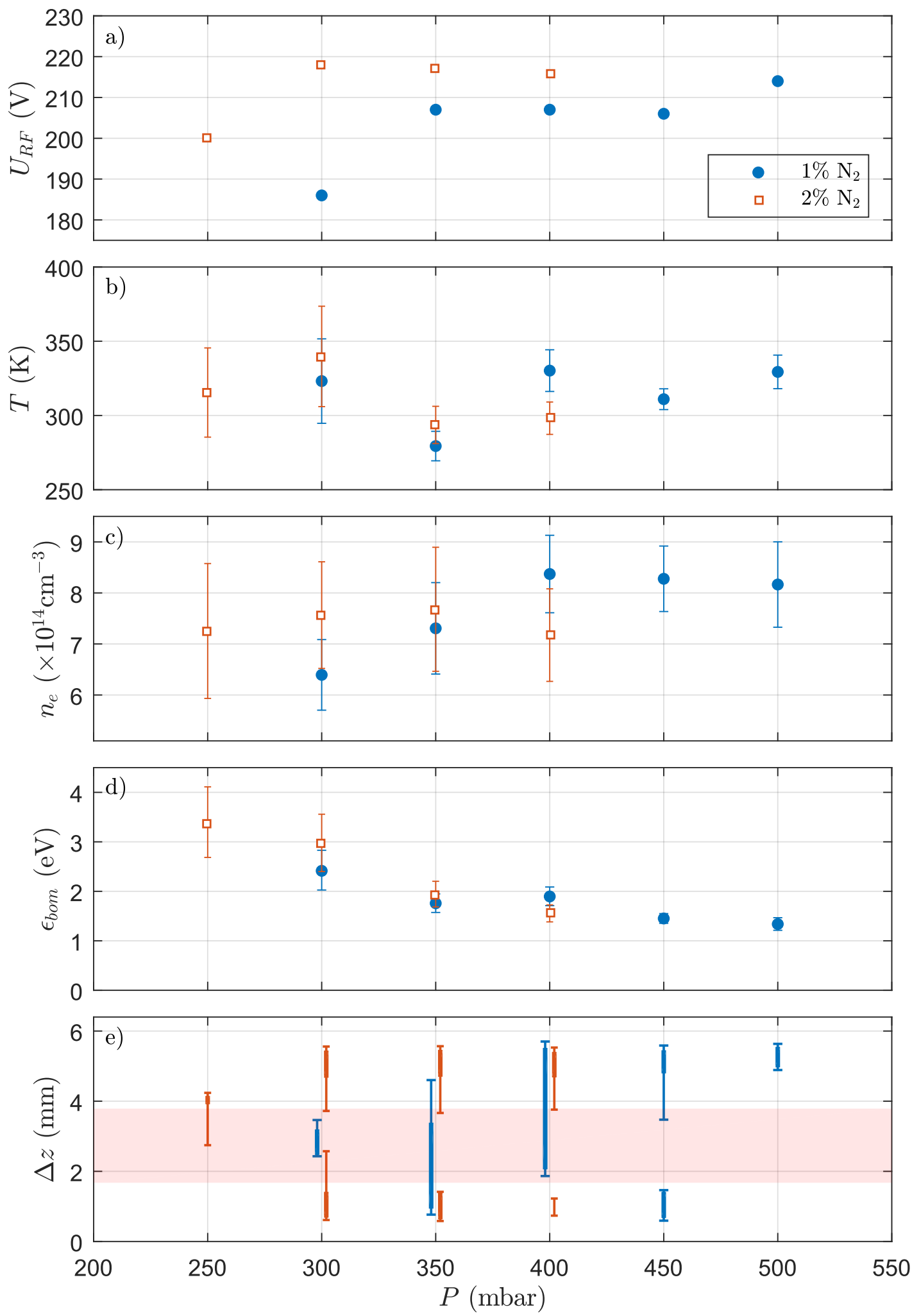

Figure 8. Measurements to determine the average ion bombardment energy with a He: $\mathrm{N}_{2}$ mixture at $\Omega_{R F} / 2 \pi=23 \mathrm{MHz}$. a) Operating $U_{R F}$ values for a microplasma confined to the aperture of trap type B, for $\mathrm{N}_{2}$ concentrations of $1 \%$ (filled circles) and $2 \%$ (empty squares). b) $T$, and c) $n_{e}$, as measured from Voigt lineshapes fitted to the He I $667 \mathrm{~nm}$ and $\mathrm{H} \alpha 656 \mathrm{~nm}$ lines, using the $U_{R F}$ from (a) and the method in section 2.3. d) $\epsilon_{b o m}$ calculated from measured results in (a-c) via time-averaging of equation (20). The uncertainties in $\epsilon_{b o m}$ are propagated due to those in $T$ and $n_{e}$, determined in a similar manner to that for the pure He data. The axial spatial extent $\Delta z$ of the microplasmas in this data set is shown in e); at $P=\{300,350,400\}$ mbar the range bars are offset slightly from the exact $P$ for clarity. 
microtrap device. The parameters associated with a He microplasma, i.e. gas temperature $T$ and electron density $n_{e}$, were both determined by OES of the He I $667 \mathrm{~nm}$ and $\mathrm{H} \alpha 656 \mathrm{~nm}$ lines, without perturbing the microplasma. Mean ion bombardment energies were calculated using the measured $T$ and $n_{e}$. Our results suggest that the microplasma technique is suited to in situ selective removal of surface adsorbates from ion microtrap electrodes.

Due to the highly collisional nature of the microplasma, the mean ion energies were found to be much lower than the sputtering thresholds of both the hydrocarbons and $\mathrm{Au}$. It was found that in a $\mathrm{He}$ microplasma, $0.3 \mathrm{eV} \leqslant \epsilon_{\text {bom }}(\mathrm{He}) \leqslant 2.1 \mathrm{eV}$. Testing with a $\mathrm{He}: \mathrm{N}_{2}$ gas mixture indicated that higher ion energies in the range $1.2 \mathrm{eV} \leqslant \epsilon_{\text {bom }}(\mathrm{He}) \leqslant 4.1 \mathrm{eV}$ can be achieved. Concerning the suitability of these microplasmas as a technique for the selective removal of surface adsorbates from ion microtrap electrodes, it will be reliant on the high energy tail of the energy distribution. Estimated surface processing times were calculated and showed that cleaning times down to $\sim 40$ minutes for type A microtrap chips should be sufficient for removal of two hydrocarbon monolayers. For trap type B, corresponding times were estimated to be $60 \mathrm{~s}$. Under operating parameters for these results, the microplasma extent across all trapping zone electrodes was almost complete for type A and complete for type B. For He: $\mathrm{N}_{2}$ mixtures under the same electrode coverage condition, the estimated cleaning time was in the range $43 \mathrm{~s} \leqslant t_{\text {clean }} \leqslant 74 \mathrm{~s}$. During these times, the sputtering of $\mathrm{Au}$ from the electrode surface is expected to be negligible, which was confirmed by optical microscopy.

The principle of the microplasma technique presented here is widely applicable to other ion traps with a 3D electrode geometry, both micro-structured and otherwise. Furthermore, it is possible that the general principle could also be applied to microtraps with a 2D electrode geometry. In principle, the technique should be applicable to a range of metallic electrode materials.

For effective use as an ion microtrap, the device must be contained in vacuum at around $10^{-11}$ mbar. Earlier work has shown that hydrocarbons adsorb onto electrode surfaces during vacuum bakeout to reach this pressure [17]. Therefore, it is necessary to conduct the proposed microplasma surface treatment process after this bakeout, while recovering to a similarly low pressure after post-processing pump down. This will require the microplasma gas to be of the highest purity grade and be introduced via vacuum-baked gas lines, such that residual gas analysis of the evacuated chamber shows recovery of the preprocessing condition. Furthermore, it is necessary that any gas used does not react with elemental species in the vacuum. The use of an inert gas such as $\mathrm{He}$ is advantageous for such a procedure. While the aforementioned procedure is possible in principle, care will be required in order to achieve the desired cleanliness for $\sim 10^{-11}$ mbar pressure upon postprocessing pump down.

When operating the microplasma after the system bakeout to achieve $\sim 10^{-11}$ mbar, water vapour will be virtually eliminated from the vacuum chamber. Therefore, the $\mathrm{H} \alpha 656 \mathrm{~nm}$ line needed for the OES will be absent, but this is not a significant problem. The analysis presented here shows that a suitable combination of $P(\mathrm{He}), \Omega_{R F} / 2 \pi$ and $U_{R F}$ can be predetermined, so that $n_{e}$ and $T$ are well defined and the resultant $\epsilon_{\text {bom }}$ and $t_{\text {clean }}$ is known. Thus, it seems reasonable to conclude that with careful measurements characterising the procedure on an identical system, spectroscopic measurements during the actual microplasma processing step after bakeout are not necessary.

The current study shows that there is experimental evidence to suggest that a small fraction of ions and neutrals in the microplasma are sufficiently energetic to remove amorphous hydrocarbon adsorbates. In principle, it is possible to record the temporal evolution of the sputtering process via optical emission from the sputtered film, e.g. via $\mathrm{CH}(\mathrm{A}-\mathrm{X})$ spectral line at $431 \mathrm{~nm}$ [65]. However, our apparatus does not have the sensitivity to detect such emission due to the much thinner surface adsorbate film expected on our devices [66]. Further experimental work with a more complex apparatus could be done to investigate this, where the ion microtrap and microplasma system are incorporated with a surface analysis apparatus. This would permit analysis of a contaminated surface immediately before and after in situ microplasma processing without exposure to atmosphere, similar to the approach of [17]. However, the true effectiveness of the microplasma technique for its intended purpose will only become apparent with a direct comparison of trapped-ion heating rates measured before and after processing.

Previous work using energetic ion beams removed not only the hydrocarbon contamination, but also the electrode material $[17,18,25]$. The method that we describe here operates at much lower bombardment energies, and so offers the prospect for selective removal of only the surface adsorbates. The technique could be particularly useful in future studies of electric field noise, for separating the effects of surface cleanliness from any due to electrode surface order [25]. 


\section{Acknowledgements}

We are pleased to thank P. Bryant and J. Bradley (Univ. Liverpool) for informative discussions and performing an initial trial with one of our microtrap devices. We also thank P. Maunz, M. Blain and R. Halti (Sandia National Labs, Albuquerque) for discussions concerning related work in their laboratory. We are grateful to Bengt Eliasson (Univ. Strathclyde) for critical reading of the manuscript.

\section{Funding}

This work was supported by funds from the Defence Sciences and Technology Laboratories and the UK government's Department for Business, Energy and Industrial Strategy.

\section{Appendix A. Calculation of plasma parameters}

The calculations of the average particle bombardment energy, the particle flux and the cleaning time all rely on the knowledge of three plasma parameters, which themselves are dependent on the electron density $n_{e}$ and the gas temperature $T$ (i.e. those parameters measured by spectroscopy). The first of these parameters is the electron temperature $T_{e}(T)$ in the bulk of the microplasma; along with $n_{e}$, this enables the ion velocity $u_{s}\left(n_{e}, T\right)$ and ion density $n_{s}\left(n_{e}, T\right)$ at the sheath edge to be calculated. This appendix describes the analysis for determining the values of $T_{e}(T), u_{s}\left(n_{e}, T\right)$ and $n_{s}\left(n_{e}, T\right)$. Unless stated otherwise, all equations in this appendix are from [28] (with appropriate modifications to incorporate all parameters in SI units). The assumed operating regime is that of a low-frequency, capacitively-coupled discharge at high pressure and with high-voltage, collisional sheaths.

\section{Appendix A.1. Electron Temperature in a High} Pressure Discharge

In the bulk of the microplasma at high pressures, $T_{e}$ can be found by numerically solving the equation

$$
\frac{\left[K_{m i}(T) K_{i z}\left(T_{e}\right)\right]^{1 / 2}}{u_{B}\left(T_{e}\right)}=\frac{\pi}{n_{g}(T) l}
$$

where $K_{m i}$ and $n_{g}$ are functions of $T$, and $K_{i z}$ and $u_{B}$ are functions of $T_{e}$. Here $K_{m i}$ is the rate constant for ion-neutral collisions in which momentum transfer occurs. $K_{i z}$ is the ionisation rate constant, $u_{B}$ is the Bohm velocity, $n_{g}$ is the gas density and $l$ is the electrode separation. The Bohm velocity is defined by

$$
u_{B}=\left(\frac{k_{B} T_{e}}{M}\right)^{1 / 2}
$$

where $M$ denotes the ion mass.

The ion velocity in the microplasma bulk, $u_{0}$, is required to calculate $K_{m i}$ :

$$
K_{m i}=u_{0} \sigma_{H e^{+}-H e, B} .
$$

where $\sigma_{H e^{+}-H e, B}=5 \times 10^{-19} \mathrm{~m}^{2}$ is the calculated $\mathrm{He}$ ion and neutral atom collisional cross-section in the plasma bulk $[28,67]$. Due to the highly collisional nature of the discharge, the ions in the microplasma bulk are assumed to be in thermal equilibrium with the neutral atoms. Therefore, assuming a Maxwellian distribution associated with the ions,

$$
u_{0}=\left(\frac{8 k_{B} T}{\pi M}\right)^{1 / 2}
$$

and so equation (A.3) can be rewritten as

$$
K_{m i}=\sigma_{H e^{+}-H e}\left(\frac{8 k_{B} T}{\pi M}\right)^{1 / 2} .
$$

In contrast, the atom ionisation rate constant $K_{i z}$ requires knowledge of the electron velocity in the bulk of the microplasma, $u_{e}$. Similar to above, this is determined by assuming a Maxwellian distribution of electron energies, thus

$$
u_{e}=\left(\frac{8 k_{B} T_{e}}{\pi m}\right)^{1 / 2},
$$

where $m$ is the mass of the electron. Strictly speaking, the microplasma generated should have an electron energy distribution (EED) that consists of three groups $[27,53]$; a) the low energy electrons that are confined by the ambipolar potential in the microplasma bulk, b) the mid-energy electrons capable of escaping from the discharge, and c) the high-energy electrons due to accelerated secondary electrons in the sheath. Here, the high-energy tail of the distribution within the microplasma bulk can be neglected due to the short penetration depth when compared to the interelectrode distance [27]. The EED is therefore more accurately described by a bi-Maxwellian distribution. However, it should be noted that the density of the mid-energy electrons is several orders of magnitude smaller than that of the low energy electrons. We find that the error associated with the average electron energy, due to neglecting the mid-energy electrons, is small in comparison to the statistical error in fitting the spectral lines.

Using equation (A.6), $K_{i z}$ can be calculated as

$$
K_{i z}=\sigma_{0} u_{e}\left(1+\frac{2 k_{B} T_{e}}{\epsilon_{i z}}\right) \exp \left(\frac{-\epsilon_{i z}}{k_{B} T_{e}}\right) .
$$

Here $\epsilon_{i z}$ is the atom ionisation energy and $\sigma_{0}$ is a constant associated with the ionisation cross-sectional area 


$$
\sigma_{0}=\pi\left(\frac{e}{4 \pi \epsilon_{0} \epsilon_{i z}}\right)^{2},
$$

where $\epsilon_{0}$ is the permittivity of free space. For a He discharge $\epsilon_{i z}=24.6 \mathrm{eV}$.

Equations (A.2), (A.5) and (A.7) are substituted into equation (A.1), along with $n_{g}(T)$ and $l$. The resulting equation is solved numerically using a Newton-Raphson algorithm to find $T_{e}$.

\section{Appendix A.2. Ion Density at the Sheath Edge}

For a high pressure discharge, the ion density at the sheath edge $n_{s}$ can be found using $u_{0}$ and $K_{m i}$ from equations (A.4) and (A.5) respectively, in conjunction with the ambipolar diffusion coefficient $D_{a}$. At high pressures, $D_{a}$ is

$$
D_{a}=\frac{k_{B} T_{e}}{M n_{g} K_{m i}} .
$$

The quantities $u_{0}, K_{m i}, D_{a}$ and $l$ can then be substituted into the equation

$$
\frac{n_{s}}{n_{0}}=\left[1+\left(\frac{l u_{B}}{\pi D_{a}}\right)^{2}\right]^{-1 / 2},
$$

where $n_{0}$ is the ion density in the bulk of the microplasma. The quasi-neutrality condition in the bulk region of the microplasma entails that $n_{0} \simeq n_{e}$. Thus equation (A.10) can be rewritten as

$$
n_{s}\left(n_{e}, T\right) \simeq n_{e}\left[1+\left(\frac{l u_{B}(T)}{\pi D_{a}(T)}\right)^{2}\right]^{-1 / 2}
$$

where the $n_{e}$ and $T$ functional dependencies are indicated in parentheses.

\section{Appendix A.3. Ion Velocity at Sheath Edge}

The ion velocity at a collisional sheath edge, $u_{s}$, is calculated using

$$
u_{s}\left(n_{e}, T\right)=\frac{u_{B}(T)}{\left(1+\frac{\pi \lambda_{D s}\left(n_{e}, T\right)}{2 \lambda_{i}(T)}\right)^{1 / 2}},
$$

where the $n_{e}$ and $T$ dependencies are indicated in parentheses. Equations (A.2) and (17) give $u_{B}$ and the mean free path $\lambda_{i}$ respectively. The Debye length at sheath edge (in $\mathrm{m}$ ) is given by $\lambda_{D s}$

$$
\lambda_{D s}=\left(\frac{\epsilon_{0} k_{B} T_{e}}{e^{2} n_{s}}\right)^{1 / 2},
$$

where $T_{e}$ and $n_{s}$ are calculated using the methods in the previous two sections.

\section{References}

[1] Ladd T D, Jelezko F, Laflamme R, Nakamura Y, Monroe C and O'Brien J L 2010 Nature 464 4553

[2] Friedenauer A, Schmitz H, Glueckert J T, Porras D and Schätz T 2008 Nat. Phys. 4 757-761

[3] Giovannetti V, Lloyd S and Maccone L 2011 Nat. Photonics 5 222-229

[4] Chou C W, Hume D B, Koelemeij J C J, Wineland D J and Rosenband T 2010 Phys. Rev. Lett. 104 070802

[5] Blatt R and Wineland D 2008 Nature 453 1008-1015

[6] Safavi-Naini A, Rabl P, Weck P F and Sadeghpour H R 2011 Phys. Rev. A 84023412

[7] Hughes M D, Lekitsch B, Broersma J A and Hensinger W K 2011 Contemp. Phys. 52 505-529

[8] Turchette Q A, Kielpinski, King B E, Leibfried D, Meekhof D M, Myatt C J, Rowe M A, Sackett C A, Wood C S, Itano W M, Monroe C and Wineland D J 2000 Phys. Rev. A 61063418

[9] Deslauriers L, Olmschenk S, Stick D, Hensinger W K, Sterk J and Monroe C 2006 Phys. Rev. Lett. 97 103007

[10] Kumph M, Henkel C, Rabl P, Brownnutt M and Blatt R 2016 New J. Phys. 1823020

[11] Boldin I A, Kraft A and Wunderlich C 2018 Phys. Rev. Lett. 12023201

[12] Sedlacek J A, Greene A, Stuart J, McConnell R, Bruzewicz C D, Sage J M and Chiaverini J 2018 Phys. Rev. A 97020302

[13] Wineland D, Monroe C, Itano W, Leibfried D, King B and Meekhof D 1998 J. Res. Natl. Inst. Stand. Technol. 103259

[14] Labaziewicz J, Ge Y, Antohi P, Leibrandt D, Brown K R and Chuang I L 2008 Phys. Rev. Lett. 100 013001

[15] Chiaverini J and Sage J M 2014 Phys. Rev. A 89 012318

[16] Bruzewicz C D, Sage J M and Chiaverini J 2015 Phys. Rev. A 91041402

[17] Hite D A, Colombe Y, Wilson A C, Brown K R, Warring U, Jördens R, Jost J D, McKay K S, Pappas D P, Leibfried D and Wineland D J 2012 Phys. Rev. Lett. 109103001

[18] Daniilidis N, Gerber S, Bolloten G, Ramm M, Ransford A, Ulin-Avila E, Talukdar I and Häffner H 2014 Phys. Rev. B 89245435

[19] Kim E, Safavi-Naini A, Hite D A, McKay K S, Pappas D P, Weck P F and Sadeghpour H R 2017 Phys. Rev. A 95033407

[20] Wilpers G, See P, Gill P and Sinclair A G 2012 Nat. Nanotechnol. 7 572-576

[21] See P, Wilpers G, Gill P and Sinclair A G $2013 \mathrm{~J}$. Microelectromechanical Syst. 22 1180-1189

[22] Wilpers G, See P, Gill P and Sinclair A G 2013 Appl. Phys. B Lasers Opt. 111 21-28

[23] Behrisch R and Eckstein W 2007 Sputtering by Particle Bombardment (Springer)

[24] McConnell R, Bruzewicz C, Chiaverini J and Sage J 2015 Phys. Rev. A 92020302

[25] Hite D A, McKay K S, Kotler S, Leibfried D, Wineland D J and Pappas D P 2017 MRS Adv. 2 2189-2197

[26] Belostotskiy S G, Ouk T, Donnelly V M, Economou D J and Sadeghi N 2010 J. Appl. Phys. 107053305

[27] Iza F, Kim G J, Lee S M, Lee J K, Walsh J L, Zhang Y T and Kong M G 2008 Plasma Process. Ploymers 5 322-344

[28] Lieberman M A and Lichtenberg A J 2005 Principles of Plasma Discharges and Materials Processing 2nd 
ed (Wiley-Interscience)

[29] Balcon N, Aanesland a and Boswell R 2007 Plasma Sources Sci. Technol. 16 217-225

[30] Zhu X M, Walsh J L, Chen W C and Pu Y K $2012 \mathrm{~J}$. Phys. D. Appl. Phys. 45295201

[31] Sismanoglu B, Cunha C, Gomes M, Caetano R and Grigorov K 2010 Brazilian J. Phys. 40 459-463

[32] Hofmann S, van Gessel A F H, Verreycken T and Bruggeman P 2011 Plasma Sources Sci. Technol. 20065010

[33] Horvatic V, Veza D, Vadla C and Franzke J $2014 \mathrm{~J}$. Anal. At. Spectrom. 29 498-505

[34] Djurović S F o S and Konjević N F o P 2009 Plasma Sources Sci. Technol. 18035011

[35] Griem H 1964 Plasma Spectroscopy (McGraw-Hill Book Company)

[36] Gigosos M A and Cardeñoso V 1996 J. Phys. B At. Mol. Opt. Phys. 29 4795-4838

[37] Akermark T, Hultquist G and Lu Q 1996 J. Mater. Eng. Perform. 5 516-520

[38] Fridman A 2008 Plasma Chemistry (Cambridge University Press)

[39] Konjevic R and Konjevic N 1997 Spectrochim. Acta Part B 52 2077-2084

[40] Park H S, Kim S J, Joh H M, Chung T H, Bae S H and Leem S H 2010 Phys. Plasmas 17033502

[41] Drake G W F 2006 Springer Handbook of Atomic, Molecular, and Optical Physics 1st ed (New York, NY: Springer-Verlag New York)

[42] Namba S, Yamasaki T, Hane Y, Fukuhara D, Kozue K and Takiyama K 2011 J. Appl. Phys. 110073307

[43] Griem H R 1974 Spectral Line Broadening by Plasmas vol 39 (Academic Press inc.)

[44] Griem H R, Baranger M, Kolb A C and Oertel G 1962 Phys. Rev. 125 177-195

[45] NIST Atomic Spectra Database

[46] Hagelaar G J M, Kroesen G M W and Klein M H 2000 J. Appl. Phys. 88 2240-2245

[47] Mahadevan P and Magnuson G D 1968 Phys. Rev. 171 103-109

[48] Taglauer E 1990 Appl. Phys. A Solids Surfaces 51 238-251

[49] Winters H F and Sigmund P 1974 J. Appl. Phys. 45 4760-4766

[50] Winchester M R and Payling R 2004 Spectrochim. Acta - Part B At. Spectrosc. 59 607-666

[51] Ferreira N P and Büger P A 1978 Zeitschrift für Naturforsch. A 33 16-19

[52] Kotz J, Treichel P and Weaver G 2006 Chemistry and Chemical Reactivity, Enhanced Review Edition (Thomson Books/Cole)

[53] Lin L and Wang Q 2015 Plasma Chem. Plasma Process. 35 925-962

[54] Wang Q, Koleva I, Donnelly V M and Economou D J 2005 J. Phys. D. Appl. Phys. 38 1690-1697

[55] Konjević N 1999 Phys. Rep. 316 339-401

[56] Moravej M, Yang X, Nowling G R, Chang J P, Hicks R F and Babayan S E 2004 J. Appl. Phys. 96 70117017

[57] García-Rosales C, Eckstein W and Roth J 1995 J. Nucl. Mater. 218 8-17

[58] Jacob W, Hopf C and Schlüter M 2006 Phys. Scr. T124 32-36

[59] Ito T and Cappelli M A 2007 Energetic neutral particle production in the cathode sheath of direct-current discharges $I S P C$-18 (Kyoto, Japan) pp 4-7

[60] Pitchford L C, Wang J, Piscitelli D and Boeuf J P 2006 IEEE Trans. Plasma Sci. 34 351-359

[61] Hsieh J H and Li C 2003 Jpn. J. Appl. Phys. 42 52955298
[62] Mason R and Pichilingi M 1994 J. Phys. D. Appl. Phys. 272363

[63] Brandenburg, R; Wagner, H, Michel, P, Trunec, D, Stahel P 2003 Diffuse dielectric barrier discharges in nitrogen-containing gas mixtures Int. Conf. Phenom. Ioniz. Gases [26th] Vol. 4 (Greifswald,)

[64] Tschiersch R, Bogaczyk M and Wagner H E $2014 \mathrm{~J}$. Phys. D. Appl. Phys. 47365204

[65] Hansen T A R, Colsters P G J, Weber J W, van de Sanden M C M and Engeln R 2009 Amorphous hydrogenated carbon etching with a plasma jet ISPC- 19 pp $2-5$

[66] Mangolini F, McClimon J B, Rose F and Carpick R W 2014 Anal. Chem. 86 12258-12265

[67] Rapp D and Francis W E 1962 J. Chem. Phys. 37 2631-2645 